\title{
Recovery of the global magnetic field configuration of 78 Virginis from Stokes IQUV line profiles
}

\author{
V. R. Khalack ${ }^{1,2}$ and G. A. Wade ${ }^{3}$ \\ 1 Département de Physique et d'Astronomie, Université de Moncton, Moncton, N.-B., E1A 3E9, Canada \\ e-mail: khalakv@umoncton.ca \\ 2 Main Astronomical Observatory, 27 Zabolotnoho Str., 03680 Kyiv, Ukraine \\ 3 Department of Physics, Royal Military College of Canada, PO Box 17000 stn "FORCES", Kingston, Ontario, K7K 4B4 Canada
}

Received 9 October 2005 / Accepted 25 January 2006

ABSTRACT

The surface magnetic field configuration of the Ap star HD 118022 (78 Vir) has been reconstructed in the framework of the magnetic charge distribution (MCD) method from the analysis of Stokes IQUV spectra obtained using the MuSiCoS spectropolarimeter at Pic du Midi Observatory. Magnetically-sensitive Fe II lines were primarily employed in the analysis, supposing that iron is evenly distributed over the stellar surface. We show that the Stokes IQUV profile shapes and variations of 78 Vir can be approximately fit assuming a global magnetic field configuration described by a slightly decentered, inclined magnetic dipole of polar surface intensity approximately $3.3 \mathrm{kG}$. The derived inclinations of the stellar rotational axis to the line of sight $i=24 \pm 5^{\circ}$ as well as to the magnetic dipole axis $\beta=124 \pm 5^{\circ}$ are in good agreement with previous estimations by other authors, whereas the sky-projected position angle ${ }^{\star}$ of the stellar rotation axis $\Omega \sim 110^{\circ}$ is reported here for the first time. In addition, several lines of $\mathrm{Cr}$ II and Ti II were studied, yielding evidence for non-uniform surface distributions of these elements, and magnetic field results similar to those derived from Fe.

Key words. stars: chemically peculiar - stars: magnetic fields - polarization - stars: individual: 78 Virginis (HD 118022)

\section{Introduction}

78 Virginis (HD 118022) is a bright Ap star, and the first star other than the sun in which magnetic field was discovered (Babcock 1947). The longitudinal magnetic field of 78 Vir, as first observed by Babcock, is variable, and constantly negative. 78 Vir is also marginally variable in broadband light, in radial velocity, and shows variations of its line profiles (Preston 1969). As suggested by Preston (1969), the observed properties of 78 Vir can be explained in the framework of the oblique rotator model (Stibbs 1950), in which the star rotates with a period of approximately 3.7 days and has a rotation axis forming a small angle with respect to the line of sight.

The first comprehensive modelling of the surface magnetic field structure of 78 Vir was performed by Borra (1980). Borra obtained high-resolution measurements of circular polarisation across the Fe II $\lambda 4520.2 \AA$ line, which he attempted to reproduce using nine different magnetic field configuration models. As argued by Borra (1980), the profiles were well reproduced with an inclined dipole geometry that contains a moderate quadrupolar component. Nevertheless, he also pointed out that a decentered $(a=0.2)$ dipole model provides essentially the same fit quality. Borra also concluded that the 78 Vir presents

* $\Omega$ increases clockwise from the axis to the North Celestial Pole and relates to the azimuth angle $\Theta$ specified by Landolfi at al. (1993) as $\Omega=360^{\circ}-\Theta$. to an observer a remarkably uniform magnetic field over most of its visible disk, at most phases. It is unfortunate that due to the small inclination of the rotational axis (about $25^{\circ}$ ) a significant part of the stellar surface remains hidden - a part which might have a less uniform field geometry.

The analysis of broadband linear polarization (BBLP) measurements of several Ap stars (including 78 Vir) by Leroy et al. (1996) suggests that the magnetic fields of many Ap stars exhibit departures from the standard oblique rotator model assuming a pure dipole field geometry. According to those authors, significant discrepancies between the BBLP observations and the "canonical model" results (Landolfi et al. 1993) required the assumption of local departures from a dipolar field. In particular, Leroy et al. (1996) showed that the BBLP variations of most stars could be reproduced assuming a dipole magnetic field geometry with slightly expanded field lines over some parts of the magnetic equator. For 78 Vir, the observed BBLP variations do not show especially strong departures from the dipolar case, although according to Leroy et al. (1996) a modified dipolar model provides a somewhat better fit to the BBLP curves for this star.

In order to better constrain the magnetic field configuration of $78 \mathrm{Vir}$, in this paper we undertake a more detailed modelling of the surface magnetic field structure based on the analysis of high resolution line profiles in all 4 Stokes parameters. In Sect. 2 we discuss the properties of obtained Stokes 
IQUV spectra, and in Sect. 3 we summarise the fundamental characteristics of 78 Vir. In Sect. 4 we describe the features of the magnetic field modelling framework, and derive the global magnetic field configuration of the star, comparing observed and computed Stokes profiles for various spectral features. In Sect. 5 we discuss the derived global magnetic field characteristics and their agreement with earlier studies.

\section{Observations}

Spectropolarimetric observations of 78 Vir were obtained in 1997 February, 1998 February and 1999 January using the $2 \mathrm{~m}$ Télescope Bernard Lyot at Observatoire du Pic du Midi (Wade et al. 2000a). The MuSiCoS cross-dispersed échelle spectrograph (Baudrand \& Böhm 1992) and dedicated polarimeter module (Donati et al. 1999) were employed for the observations.

The MuSiCoS spectrograph is a table-top instrument, which allows the acquisition of a stellar spectrum in a given polarization state (Stokes $V, Q$ or $U$ ) throughout the spectral range from 4500 to $6600 \AA$ with a resolving power of about 35000 , in a single exposure. The spectrograph is fed by a double optical fibre directly from the Cassegrain-mounted polarimeter. The optical characteristics of the polarization analyser, as well as the spectropolarimeter observing procedures, are described in detail by Donati et al. (1999). Observing details specific to the acquisition, reduction and analysis of the 78 Vir spectra are provided by Wade et al. (2000a).

The journal of spectropolarimetric observations is reported by Wade et al. (2000a). The 52 Stokes $V, Q$ and $U$ spectra, obtained on 18 different nights, cover the whole rotational period of 78 Vir approximately uniformly, and provide an average $\mathrm{S} / \mathrm{N}$ of about 370 .

\section{Fundamental parameters of 78 Vir}

78 Vir was classified by Cowley et al. (1969) as A1pCrSrEu. Adelman (1973a, 1973b) performed both a line identification and abundance analysis of this star. The distance to 78 Vir $d=48 \pm 15 \mathrm{pcs}$ and its radius $R=1.77 \pm 0.68 R_{\odot}$ are derived by Monier (1992) using the Infrared Flux Method. On the other hand, taking into account the mean angular diameter $\theta_{\mathrm{a}}=0.343$ milliarcsec obtained by Monier (1992) for this star and its distance $d=56.2 \pm 2.5$ pc derived from the Hipparcos parallax (ESA 1997), we find a somewhat larger value for the stellar radius $R=2.06 \pm 0.17 R_{\odot}$. Using the Hipparcos visual magnitude and parallax we have found the absolute visual magnitude of 78 Vir, $M_{\mathrm{v}}=1.18 \pm 0.10$. Taking into account the bolometric correction (Flower 1996), which corresponds to Monier's (1992) $9200 \pm 290 \mathrm{~K}$ effective temperature and the bolometric zeropoint correction $B C_{\mathrm{V}}=-0.07$, which is obtained assuming $M_{\text {bol }}^{\odot}=4.74$ for the Sun (Bessell et al. 1998), we can find the absolute luminosity for 78 Vir $L_{\star}=(27.3 \pm 2.5) L_{\odot}$. For 78 Vir Monier (1992) also estimates the integrated flux $f_{\star}=(2.7 \pm 0.27) \times 10^{-7} \mathrm{erg} \mathrm{sm}^{-2} \mathrm{~s}^{-1}$. Taking into account the distance to the star, this provides the luminosity $L_{\star}=(26.5 \pm 2.8) L_{\odot}$. Finally, employing the stellar radius and the effective temperature (see Table 1) we obtain a third
Table 1. Fundamental parameters of 78 Vir.

\begin{tabular}{lcc}
\hline \hline Parameter & Value & Reference \\
\hline Spectral type & A1pCrSrEu & Cowley et al. (1969) \\
Age & $3.0_{-1.3}^{+0.7} \times 10^{8} \mathrm{y}$ & this work \\
Distance & $56.2 \pm 2.5 \mathrm{pcs}$ & Hipparcos (ESA 1997) \\
Period & $3.7220 \pm 0.003$ & Preston (1969) \\
$T_{\text {eff }}$ & $9200 \pm 290 \mathrm{~K}$ & Monier (1992) \\
$\log g$ & $4.50 \pm 0.25$ & ibidem \\
$M_{\mathrm{v}}$ & $1.18 \pm 0.10$ & this work \\
$L_{\star}$ & $27.3 \pm 2.5 L_{\odot}$ & this work \\
$R_{\star}$ & $2.06 \pm 0.17 R_{\odot}$ & this work \\
$M_{\star}$ & $2.18 \pm 0.06 M_{\odot}$ & this work \\
$V_{\mathrm{e}} \sin i$ & $12 \pm 1 \mathrm{~km} \mathrm{~s}^{-1}$ & this work \\
$i$ & $25^{\circ} \pm 5^{\circ}$ & Leroy et al. (1996) \\
$\beta$ & $120^{\circ} \pm 5^{\circ}$ & ibidem \\
\hline
\end{tabular}

estimate of the absolute luminosity $L_{\star}=27.4 \pm 4.5 L_{\odot}$. All of these values are in good mutual agreement. The luminosity $L_{\star}=(27.3 \pm 2.5) L_{\odot}$ together with the effective temperature (see Table 1) allow us to find the stellar mass $M_{\star}=2.18 \pm$ $0.06 M_{\odot}$ and age $3.0_{-1.3}^{+0.7} \times 10^{8}$ years (see Fig. 1 ) by spline interpolation (Sandwell 1987) in the model evolutionary tracks of Schaller et al. (1992) for metallicity $Z=0.02$. The derived age suggests that 78 Vir has completed approximately $37 \%$ of its main sequence life. The mass and radius provide $\log g=4.16 \pm 0.07$. This value is marginally inconsistent with that of the Monier (1992), but it is in good agreement with the value $\log g=4.20$ reported by King et al. (2003). A recent investigation of the Ursa Major stream characteristics performed by King et al. (2003) derived $Z=0.016-0.02$ and age $(5 \pm 1) \times 10^{8}$ years for the stream. They found that $78 \mathrm{Vir}$ is a "certain member" of the stream from photometric data, while the kinematic characteristics of the star argue for its "probable non-membership". The derived age for $78 \mathrm{Vir}$ is marginally inconsistent with the UMa stream age. The published metallicity of $78 \mathrm{Vir}$ is $\log (\mathrm{Fe} / \mathrm{H})_{\star}-\log (\mathrm{Fe} / \mathrm{H})_{\odot}=_{+1.58}^{-0.13}$ (Cayrel de Strobel et al. 1997) and it is not clear if this star truly has a solar metallicity. For this reason the derived uncertainties of the mass and age may be somewhat larger than indicated here.

Babcock (1947) observed that the longitudinal magnetic field of 78 Vir was variable, but always negative. Analysing Babcock's data along with his own measurements, Preston (1969) determined the periodic character of the magnetic field variability and derived the ephemeris:

$\mathrm{JD}($ magnetic maximum $)=2434816.9+3 \mathrm{~d} 7220 \cdot E$.

Several authors have confirmed this period on the basis of further magnetic field measurements (Wolff \& Wolff 1971; Wolff \& Bonsack 1972; Wolff 1978; Borra 1980; Borra \& Landstreet 1980; Landstreet 1982; Wade et al. 2000b), while Landstreet (private communication) has estimated the accuracy of the period determination to be \pm 0.003 . Preston (1969) also found that the crossover effect is very pronounced and is present throughout most of the magnetic cycle. This feature is particularly remarkable at phase 0.85 (Wolff \& Bonsack 1972; see also Wade et al. 2000a). 


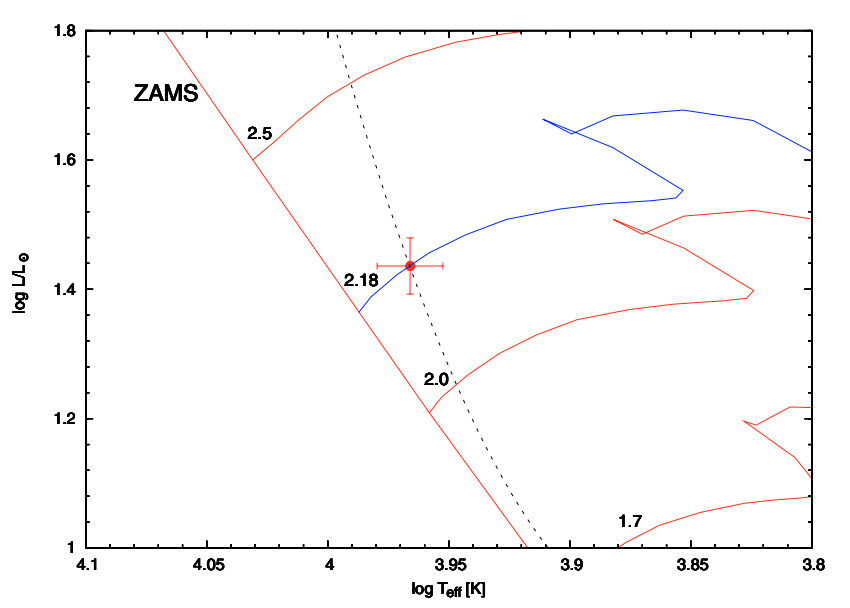

Fig. 1. The position of 78 Vir on the H-R diagram is compared with the theoretical evolutionary tracks of Schaller et al. (1992), computed for solar metallicity and masses $M=1.7,2.0$ and $2.5 M_{\odot}$. The age $3.0 \times 10^{8}$ years and the mass $M_{\star}=2.18 M_{\odot}$ of the star are estimated by interpolation of the theoretical evolutionary tracks. The dashed line shows the isochrone, which corresponds to the age of $78 \mathrm{Vir}$ $\left(3.0 \times 10^{8} \mathrm{y}\right)$.

Applying the oblique rotator model with the distorted dipole approximation, Leroy (private communication) found that they must adopt a period 3.7218 days in order to fit the observed BBLP variations. As discussed in Sect. 1, their model, using local modifications of the axisymmetric magnetic field imposed near the magnetic equator, differs only mildly from a dipole configuration. The derived rotational axis inclination and dipole obliquity $i=25^{\circ}$ and $\beta=120^{\circ}$ provide a good match to the longitudinal field and linear polarisation variations. Nevertheless, the period inferred is somewhat shorter than that obtained by other authors (Eq. (1)), and Wade et al. (2000b) reported that this period is only barely consistent with the available longitudinal field measurements.

78 Vir also shows photometric variability in the visible and the infrared with the same period (Eq. (1)). Combining the visual flux variability in the $u v b y$ bands, obtained by Wolff \& Wolff (1971), with their own new observations, Catalano \& Leone (1994) have tried to improve the period determination. Their analysis results in the new ephemeris

$\mathrm{JD}(\mathrm{y}$ min. $)=2434816+(3.722084 \pm 0.000042) E$,

which was recently confirmed by Leone \& Catanzaro (2001). These authors have shown with the help of Hipparcos photometry that the light variations of 78 Vir are not purely sinusoidal. Taking into account all archival magnetic field observations as well as their own data, Leone \& Catanzaro (2001) have shown that the longitudinal field measurements are in phase only when the 3.722084 day period is adopted. Moreover, by adopting the 3.7218 day period suggested by Leroy (private communication), they find a 0.12-cycle phase shift between the Hipparcos light curves and the other light curves.

Monier (1992) has derived for 78 Vir from the simulation of the energy distribution in the spectral range from $1200 \AA$ to $22000 \AA$ an effective temperature $T_{\text {eff }}=9200 \pm 290 \mathrm{~K}$, a surface gravity $\log g=4.5$ and a photospheric metallicity
$[\mathrm{M} / \mathrm{H}]=10$. In addition, using a model atmosphere with these values of $\log g$ and $[\mathrm{M} / \mathrm{H}]$, he has found that the effective temperature $T_{\text {eff }}=9300 \mathrm{~K}$ provides the best description of the spectral energy distribution in the region from $1200 \AA$ to $8000 \AA$ at rotational phase 0.0 , while $T_{\text {eff }}=9200 \mathrm{~K}$ seems to be the best effective temperature, at phase 0.5 . These temperatures are somewhat lower than most previous estimates, ranging from $9700 \mathrm{~K}$ to $10700 \mathrm{~K}$ (Mihalas \& Henshaw 1966; Wolff 1967; Jugaku \& Sargent 1968), probably because the models used by these authors were unblanketed and calculated for solar abundances.

The fundamental parameters of 78 Vir are summarised in Table 1.

\section{Modelling of the Stokes IQUV spectra}

According to the discussion of Sect. 3, an ATLAS9 model (Kurucz 1994) with parameters $T_{\text {eff }}=9250 \mathrm{~K}, \log g=4.5$, $[\mathrm{M} / \mathrm{H}]=0$ and microturbulent velocity $v_{\mathrm{t}}=0 \mathrm{~km} \mathrm{~s}^{-1}$ successfully approximates the stellar atmosphere of $78 \mathrm{Vir}$.

We describe the structure of the surface magnetic field of 78 Vir (assumed to be a rigidly rotating and spherically symmetric star) using the magnetic charge distribution (MCD) method (Gerth et al. 1997; Khalack et al. 2001). Originally, the MCD method considered a system of spatially separated point field sources with virtual magnetic charges in the stellar interior. In order to provide zero magnetic flux through the stellar surface the sum of "magnetic charges" should be kept to zero. These sources produce a magnetic field whose potential at each point on the stellar surface is specified by the superposition of potentials of individual sources (Gerth et al. 1997; Gerth \& Glagolevskij 2001, 2004). Since the number of sources is usually more than one, we actually operate with a system of several magnetic dipoles located in the stellar interior. In order to minimize the number of free model parameters, the most convenient way is to consider a system of two sources, which mathematically formulate a magnetic dipole (Khalack et al. 2001). When the dipole is centered on the stellar centre and the dipole size is much smaller than the stellar radius (Khalack 2002), the MCD model transforms to the conventional model of a symmetric inclined magnetic rotator (Stibbs 1950). Otherwise, we deal with a more complex magnetic field configuration. The mathematical verification of the MCD model as well as the procedure of specification of the angle $\beta$ between the rotational and magnetic dipole axes, the coordinates and the field strengths of the positive and negative magnetic poles on the basis of the derived free model parameters is described in detail by Khalack et al. (2003).

\subsection{Procedure}

From previous analyses of the Stokes IQUV spectra of 78 Vir, it has been found by Wade et al. (2000a) that the three strong Fe II lines $\lambda 4923.93 \AA, \lambda 5018.44 \AA$ and $\lambda 5169.03 \AA$ show the strongest Zeeman signatures of any lines in the optical spectra of CP stars. The real magnetic field structure of $78 \mathrm{Vir}$ is expected to differ only marginally from a centered magnetic dipole (based on previous modeling efforts: Borra 1980). 
The most attention is therefore paid to the variability of the Stokes $I$ and $V$ profiles, which contain the most information about the global surface magnetic field configuration. The Stokes $I$ variation provides information about the surface distribution of the chemical abundance, as well as the stellar radial velocity $V_{\mathrm{r}}$ and projected rotational velocity $V_{\mathrm{e}} \sin i$. The Stokes $V$ profiles are sensitive to the global field configuration, in particular the lower-order multipolar components. The Stokes $Q$ and $U$ profiles also provide some constraint on the global field morphology, but are most sensitive to the smaller-scale structure of the field. Unfortunately, the available Stokes $Q$ and $U$ profiles have low relative $\mathrm{S} / \mathrm{N}$ ratio (Wade et al. 2000a), and in this study they are used primarily to check the results of the Stokes $I$ and $V$ profile simulations and to determine the sky-projected position angle of the rotational axis, $\Omega$. According to Khalack et al. $(2001,2003)$ this angle $\left(0 \leq \Omega<360^{\circ}\right)$ is counted clockwise from the rotation axis to the North Celestial Pole in the plane of the sky, and is related to the azimuth angle $\Theta$ specified by Landolfi et al. (1993) by $\Omega=360^{\circ}-\Theta$. The opposite counting of the position angle in the MCD model is compensated for by the negative sign in definition of the $B_{\mathrm{y}}$-component of the local magnetic field. Hence a right-handed reference frame is applicable.

We have examined the spectra for additional lines with prominent polarisation signatures, in order to compile a list of lines that are especially appropriate for this task. To perform the line identification we use the VALD-2 resources (Kupka et al. 1999; Ryabchikova et al. 1999). The adopted list contains Fe II, Fe I, Cr II, CrI, Ti II, Ti I and Mg II lines, but for the present study we will concentrate primarily on the strongest Fe II lines. The main reason for this is that Fe is presumably almost uniformly distributed over the stellar surface (see Sect. 4.2) and in this way we exclude from the fitting procedure a model of the surface abundance distribution. Table 2 presents the final list of Fe II lines used in the simulation procedure, together with lines of some other elements that are responsible for blends.

Some of the Fe II lines listed in Table 2 have no detectable features in the Stokes $Q$ and $U$ spectra. Nevertheless, they are comparatively strong lines (in the Stokes $I$ spectra) with clear variability in the Stokes $V$ spectra, and are analysed without the linear polarization data in order to check our final results.

All line profiles are simulated using the ZEEMAN2 polarised spectrum synthesis code (Landstreet 1988; Wade et al. 2001). The code has been modified to include a magnetic field described within the framework of the MCD method (Khalack et al. 2003), and to allow for an automatic minimization of the model parameters using the downhill simplex method (Press et al. 1992). The relatively poor efficiency of the downhill simplex method, requiring a large number of function evaluations, is a well-known problem. Repeating the minimization routine $3 \div 4$ times in the vicinity of a supposed minimum in the parameter space allows us to check if the method converges to a global minimum. In our case, this technique requires a comparatively long computational time due to the large amount of analysed observational data and the large number of free parameters.

The free model parameters employed in the line profile simulation can be divided into two groups: those that describe
Table 2. Here the individual columns specify the ion, the wavelength, the quantum number $J$ and the Lande factor for lower and upper atomic levels, the observed Stokes $I$ profile depth and the line scaling factor (LSF) (see Sect. 4.3), derived from the comparison of simulated equivalent width of the Stokes $Q$ and $U$ Zeeman profiles with the BBLP data (Leroy 1995). Asterisks marks the LSF calculated from Stokes $Q$ and $U$ profiles simulated for a field structure that is derived from only Stokes $I$ and $V$ profile variability (see Table 3).

\begin{tabular}{lccccccl}
\hline \hline Ion & $\lambda, \AA$ & $J_{\text {lo }}$ & $g_{\text {lo }}$ & $J_{\text {up }}$ & $g_{\text {up }}$ & $I_{\text {obs }}$ & LSF \\
\hline Fe II & 4620.52 & 3.5 & 1.21 & 3.5 & 1.40 & 0.33 & 0.035 \\
Fe II & 4635.32 & 2.5 & 1.20 & 3.5 & 1.13 & 0.32 & $0.036^{*}$ \\
Fe I & 4635.85 & 1.0 & 1.49 & 2.0 & 1.89 & & \\
Fe II & 4923.93 & 2.5 & 2.00 & 1.5 & 2.40 & 0.58 & 0.079 \\
Ti I & 5017.95 & 4.0 & 1.50 & 5.0 & 1.18 & & \\
Cr I & 5018.15 & 2.0 & 1.16 & 3.0 & 1.09 & & \\
Fe II & 5018.44 & 2.5 & 2.00 & 2.5 & 1.87 & 0.60 & 0.102 \\
Cr II & 5018.84 & 3.5 & 1.24 & 3.5 & 1.42 & & \\
Fe II & 5100.61 & 4.5 & 1.54 & 4.5 & 1.34 & & \\
Fe II & 5100.66 & 4.5 & 1.31 & 3.5 & 1.40 & & \\
Fe II & 5100.73 & 4.5 & 1.54 & 5.5 & 1.36 & & \\
Fe II & 5100.85 & 1.5 & 0.80 & 1.5 & 0.72 & 0.47 & $0.083^{*}$ \\
Fe I & 5168.90 & 3.0 & 1.50 & 3.0 & 1.75 & & \\
Fe II & 5169.03 & 2.5 & 2.00 & 3.5 & 1.70 & 0.59 & 0.178 \\
Fe I & 5169.30 & 4.0 & 1.26 & 3.0 & 1.32 & & \\
Fe II & 5197.48 & 2.5 & 1.20 & 1.5 & 0.72 & & \\
Fe II & 5197.58 & 2.5 & 0.57 & 1.5 & 0.44 & 0.39 & 0.073 \\
Fe II & 5362.74 & 3.5 & 1.15 & 4.5 & 1.24 & & \\
Fe II & 5362.87 & 4.5 & 1.15 & 3.5 & 1.40 & 0.45 & $0.112^{*}$ \\
Cr I & 5362.96 & 3.0 & 1.33 & 3.0 & 1.06 & & \\
Fe II & 5362.97 & 3.5 & 1.44 & 4.5 & 1.34 & & \\
Cr II & 5363.88 & 3.5 & 1.71 & 3.5 & 1.39 & & \\
Fe II & 6247.35 & 1.5 & 0.40 & 2.5 & 0.62 & & \\
Fe II & 6247.56 & 2.5 & 1.33 & 1.5 & 1.72 & 0.36 & $0.036^{*}$ \\
Fe II & 6432.68 & 2.5 & 2.00 & 2.5 & 1.65 & 0.24 & 0.026 \\
Fe II & 6516.08 & 2.5 & 2.00 & 3.5 & 1.58 & 0.25 & 0.032 \\
\hline
\end{tabular}

the magnetic field structure, and those that describe the stellar geometry and atmospheric characteristics. The first group includes the parameters $Q_{\mathrm{r}}, a_{1}$ and $a_{2}$ (which specify the modulus of "magnetic charges" and their distance from the center of the star, expressed in the units of stellar radius), and the parameters $\lambda_{1}, \delta_{1}$ and $\lambda_{2}, \delta_{2}$ (which specify the spherical coordinates of the "charges" in the rotational reference frame of the star). Meanwhile, the second group includes the parameters $\Omega$ (the position angle), $i$ (the rotational axis inclination with respect to the line of sight), $V_{\mathrm{r}}$ (the heliocentric radial velocity), $V_{\mathrm{e}} \sin i$ (the projected rotational velocity), and $\log \left(N_{\mathrm{x}} / N_{\text {tot }}\right)$ (the abundance(s) of the element(s) forming the line to be modeled). Instead of $V_{\mathrm{e}} \sin i$ we can obviously use only $V_{\mathrm{e}}$ as a free parameter, but $V_{\mathrm{e}} \sin i$ is preferable here, because it allows us to compare our estimate of this parameter directly with the results of other authors. The minimum number of free model parameters is 11 for the MCD model if we do not take into account the linear polarization data, and 12 if we do. Sometimes, to obtain a good fit to the line profiles we need to include in the simulation lines of other chemical elements (see Table 2). In this case the number of free model parameters grows with the number 
Table 3. Results of Fe II lines simulation for $T_{\text {eff }}=9250 \mathrm{~K}, \log g=4.5$ and $v_{\mathrm{t}}=0 \mathrm{~km} \mathrm{~s}^{-1}$. The first column indicates the free parameters, while the other columns specify the parameter values for the given line profile. The last column provides the averaged estimation errors for each parameter. The first 6 rows show the fit quality for the each analysed Stokes profile (Eq. (3)), weighted and unweighted (Eqs. (4)-(6)) $\chi^{2}$-function. The following 12 rows show the best fit values of the free model parameters, while the final 9 rows provide the characteristics of the magnetic dipole poles, which are derived from the free parameters.

\begin{tabular}{|c|c|c|c|c|c|c|c|c|c|c|c|c|}
\hline Line, Å & 4620 & 4635 & 4923 & 5018 & 5100 & 5169 & 5197 & 5362 & 6247 & 6432 & 6516 & $\sigma_{\mathrm{er}}$ \\
\hline$\chi_{I}^{2}$ & 2.92 & 10.91 & 8.96 & 6.97 & 7.40 & 13.10 & 16.06 & 5.54 & 2.27 & 8.83 & 5.94 & \\
\hline $4 \chi_{V}^{2}$ & 6.14 & 7.86 & 9.14 & 8.69 & 6.97 & 9.09 & 8.71 & 6.64 & 5.00 & 12.24 & 7.39 & \\
\hline $6 \chi_{Q}^{2}$ & 7.75 & - & 7.73 & 5.76 & - & 8.23 & 6.35 & - & - & 12.15 & 9.30 & \\
\hline $6 \chi_{U}^{2}$ & 7.42 & - & 10.04 & 6.82 & - & 10.79 & 6.80 & - & - & 9.99 & 6.19 & \\
\hline$\chi_{\mathrm{w}}^{2}$ & 6.06 & 9.39 & 8.97 & 7.06 & 7.19 & 10.30 & 9.45 & 6.09 & 3.64 & 10.81 & 7.20 & \\
\hline$\chi^{2}$ & 1.75 & 6.44 & 3.55 & 2.81 & 4.57 & 4.64 & 5.11 & 3.60 & 1.76 & 3.90 & 2.59 & \\
\hline $\mathrm{Qr}, \mathrm{kG}$ & 180 & 254 & 303 & 202 & 230 & 221 & 151 & 192 & 398 & 173 & 289 & 50 \\
\hline$a_{1}, 10^{-3}$ & 8.4 & 6.7 & 4.3 & 6.7 & 5.3 & 7.3 & 12.6 & 9.7 & 5.4 & 8.9 & 6.4 & 1.2 \\
\hline$\lambda_{1}$ & $13^{\circ}$ & $24^{\circ}$ & $34^{\circ}$ & $41^{\circ}$ & $33^{\circ}$ & $15^{\circ}$ & $-4^{\circ}$ & $19^{\circ}$ & $69^{\circ}$ & $23^{\circ}$ & $20^{\circ}$ & $5^{\circ}$ \\
\hline$\delta_{1}$ & $-45^{\circ}$ & $-46^{\circ}$ & $-52^{\circ}$ & $-51^{\circ}$ & $-60^{\circ}$ & $-30^{\circ}$ & $-40^{\circ}$ & $-44^{\circ}$ & $-39^{\circ}$ & $-45^{\circ}$ & $-39^{\circ}$ & $8^{\circ}$ \\
\hline$a_{2}, 10^{-3}$ & 3.1 & 3.7 & 3.5 & 5.2 & 4.3 & 2.5 & 1.6 & 3.7 & 5.1 & 3.8 & 2.7 & 0.3 \\
\hline$\lambda_{2}$ & $118^{\circ}$ & $125^{\circ}$ & $144^{\circ}$ & $135^{\circ}$ & $145^{\circ}$ & $85^{\circ}$ & $111^{\circ}$ & $101^{\circ}$ & $113^{\circ}$ & $96^{\circ}$ & $111^{\circ}$ & $10^{\circ}$ \\
\hline$\delta_{2}$ & $-16^{\circ}$ & $-9^{\circ}$ & $-9^{\circ}$ & $-14^{\circ}$ & $-12^{\circ}$ & $-11^{\circ}$ & $-11^{\circ}$ & $-26^{\circ}$ & $-11^{\circ}$ & $-27^{\circ}$ & $-11^{\circ}$ & $10^{\circ}$ \\
\hline$\Omega$ & $109^{\circ}$ & - & $109^{\circ}$ & $109^{\circ}$ & - & $109^{\circ}$ & $102^{\circ}$ & - & - & $164^{\circ}$ & $128^{\circ}$ & $17^{\circ}$ \\
\hline$i$ & $22^{\circ}$ & $24^{\circ}$ & $22^{\circ}$ & $23^{\circ}$ & $25^{\circ}$ & $21^{\circ}$ & $29^{\circ}$ & $27^{\circ}$ & $26^{\circ}$ & $29^{\circ}$ & $24^{\circ}$ & $5^{\circ}$ \\
\hline $\log \left(\mathrm{Fe} / N_{\mathrm{tot}}\right)$ & -3.36 & -2.82 & -3.26 & -3.24 & -3.20 & -3.12 & -3.25 & -2.86 & -3.17 & -2.97 & -3.50 & 0.22 \\
\hline$V_{\mathrm{r}}$ & -8.78 & -8.42 & -7.82 & -7.51 & -8.69 & -7.23 & -7.93 & -8.25 & -7.99 & -6.57 & -6.74 & 1.18 \\
\hline$V_{\mathrm{e}} \sin i$ & 10.9 & 11.1 & 11.9 & 12.0 & 11.5 & 12.6 & 11.9 & 13.0 & 11.9 & 13.7 & 11.5 & 0.9 \\
\hline$\beta$ & $125^{\circ}$ & $124^{\circ}$ & $122^{\circ}$ & $120^{\circ}$ & $123^{\circ}$ & $118^{\circ}$ & $129^{\circ}$ & $126^{\circ}$ & $123^{\circ}$ & $126^{\circ}$ & $122^{\circ}$ & $5^{\circ}$ \\
\hline$a_{0}, 10^{-3}$ & 4.5 & 3.9 & 2.9 & 6.0 & 3.4 & 4.3 & 6.5 & 5.8 & 4.8 & 5.6 & 3.6 & 1.6 \\
\hline$a, 10^{-3}$ & 4.5 & 3.8 & 2.6 & 0.7 & 3.4 & 3.4 & 6.2 & 4.4 & 2.1 & 3.8 & 3.4 & 1.6 \\
\hline$B_{\mathrm{p}}, \mathrm{kG}$ & 3.25 & 3.95 & 3.47 & 3.18 & 3.13 & 3.02 & 4.00 & 3.45 & 3.42 & 2.69 & 3.92 & 0.7 \\
\hline$\lambda_{\mathrm{p}}$ & $-9^{\circ}$ & $-10^{\circ}$ & $-6^{\circ}$ & $-7^{\circ}$ & $-10^{\circ}$ & $-8^{\circ}$ & $-11^{\circ}$ & $-8^{\circ}$ & $-11^{\circ}$ & $-8^{\circ}$ & $-8^{\circ}$ & $5^{\circ}$ \\
\hline$\delta_{\mathrm{p}}$ & $-35^{\circ}$ & $-33^{\circ}$ & $-30^{\circ}$ & $-30^{\circ}$ & $-33^{\circ}$ & $-28^{\circ}$ & $-37^{\circ}$ & $-36^{\circ}$ & $-34^{\circ}$ & $-36^{\circ}$ & $-32^{\circ}$ & $12^{\circ}$ \\
\hline$B_{\mathrm{n}}, \mathrm{kG}$ & -3.18 & -3.82 & -3.46 & -3.16 & -3.12 & -2.95 & -3.85 & -3.36 & -3.41 & -2.62 & -3.86 & 0.7 \\
\hline$\lambda_{\mathrm{n}}$ & $170^{\circ}$ & $170^{\circ}$ & $174^{\circ}$ & $173^{\circ}$ & $170^{\circ}$ & $172^{\circ}$ & $168^{\circ}$ & $171^{\circ}$ & $169^{\circ}$ & $171^{\circ}$ & $172^{\circ}$ & $5^{\circ}$ \\
\hline$\delta_{\mathrm{n}}$ & $35^{\circ}$ & $33^{\circ}$ & $30^{\circ}$ & $30^{\circ}$ & $33^{\circ}$ & $28^{\circ}$ & $37^{\circ}$ & $35^{\circ}$ & $33^{\circ}$ & $36^{\circ}$ & $32^{\circ}$ & $12^{\circ}$ \\
\hline
\end{tabular}

of chemical elements under investigation, but usually does not exceed 15 parameters.

Initially we specify arbitrarily the values of the free model parameters in the range of their possible values and simulate the Stokes $I Q U V$ spectra at the phases of the observations. We then compare the simulated profiles with the observed spectra, and calculate the reduced $\chi^{2}$, which we adopt as a measure of the fit quality. The expression for the $\chi^{2}$ function, which reflects the agreement between (for example) the simulated Stokes $I$ $\left(I_{\lambda_{j}}\left(\varphi_{i}\right)\right)$ and observed Stokes $I\left(I_{i, \lambda_{j}}^{\mathrm{obs}}\right)$ spectra is given by:

$\chi_{I}^{2}=\frac{1}{N_{I}} \sum_{i=1}^{N_{I}} \frac{1}{N_{i}} \sum_{j=1}^{N_{i}}\left(\frac{I_{i, \lambda_{j}}^{\mathrm{obs}}-I_{\lambda_{j}}\left(\varphi_{i}\right)}{\sigma\left[I_{i, \lambda_{j}}^{\mathrm{obs}}\right]}\right)^{2}$,

where $\sigma\left[I_{i, \lambda_{j}}^{\text {obs }}\right]$ corresponds to the measurement errors, $\varphi_{i}$ is the rotational phase of the observation, $N_{I}$ specifies the number of spectra, while $N_{i}$ is the number of pixels in each analysed line profile. In fact, although the simulated spectra are calculated with approximately the same resolving power as the observed spectra, this does not provide a direct coincidence of wavelengths in the simulated and observed spectra. Therefore, during the $\chi^{2}$ function evaluation the simulated spectral intensity at the exact observed wavelength is calculated using a linear interpolation.
The scale of variability and the measurement errors are different for each of the four Stokes IQUV spectra (Wade et al. 2000a). In order to balance (from a statistical point of view) the information flow from each of the four Stokes spectra, the respective $\chi^{2}$ contributions are weighted.

The weights are derived from the comparison of the best fit values of $\chi_{V}^{2}, \chi_{Q}^{2}, \chi_{U}^{2}$ with $\chi_{I}^{2}$ (when the ordinary $\chi^{2}$ function is minimized) and provide approximately the same weighted value of these functions for the majority of analysed lines (see Table 3). The weighted $\chi_{\mathrm{w}}^{2}$ functions is specified in the following way:

$\chi_{\mathrm{w}}^{2}=\frac{1}{4}\left[\chi_{I}^{2}+4 \chi_{V}^{2}+6\left(\chi_{Q}^{2}+\chi_{U}^{2}\right)\right]$

or

$\chi_{\mathrm{w}}^{2}=\frac{1}{2}\left[\chi_{I}^{2}+4 \chi_{V}^{2}\right]$

if we work only with the Stokes $I$ and $V$ spectra. The value of the $\chi_{\mathrm{w}}^{2}$ function is reduced throughout the minimization procedure, varying the values of all free model parameters. When it reaches its global minimum, we can also calculate the ordinary $\chi^{2}$ function:

$\chi^{2}=\frac{1}{4}\left[\chi_{I}^{2}+\chi_{V}^{2}+\chi_{Q}^{2}+\chi_{U}^{2}\right]$ 
We begin by simulating the strong Fe II $\lambda 4923.927 \AA, \lambda 5018.44$ and $\lambda 5169.03$ lines, for which the minimization process has been repeated for $13 \div 15$ times starting from different locations in the free model parameter space in order to avoid local minima. In the case of $78 \mathrm{Vir}$, the downhill simplex method converges to four global minima, which are caused by the decentered dipole model symmetry in the MCD method. Two global minima correspond to the parameter sets $i, \beta, \Omega$ and $i, \beta, 180^{\circ}+\Omega$ which differ only by the angle $\Omega$ and provide exactly the same value of the $\chi_{\mathrm{w}}^{2}$-function. Models with the opposite direction of stellar rotation $\left(180^{\circ}-i, 180^{\circ}-\beta, \Omega\right.$ or $180^{\circ}-i, 180^{\circ}-\beta, 180^{\circ}+\Omega$ ) do not result in minima. The other configurations $\left(i, 180^{\circ}-\beta, \Omega\right.$ and $\left.i, 180^{\circ}-\beta, 180^{\circ}+\Omega\right)$ correspond to models in which the positive magnetic pole faces the observer. These configurations are not applicable to the case of $78 \mathrm{Vir}$, where we always see the negative magnetic pole, while the positive pole is partially visible just for phases close to $\varphi=0.0$ (Babcock 1947; Borra 1980). The other two global minima have parameter sets which differ from the previous ones by the location of the "magnetic charges". The two "magnetic charges" can be located in two different stellar hemispheres (separated by the equatorial plane), or in a single hemisphere. If they are not significantly shifted from the stellar centre, they can form the same dipole axis and will produce almost the same surface magnetic field configuration. The differences between the two models can be revealed by the sensitivity of $\chi_{\mathrm{w}}^{2}$ function on the parameter values, but the minima will still exist. Preference is given to the deepest global minimum, obtained for the model with the two "magnetic charges" located in the same stellar hemisphere. For the other lines the minimization process was performed only $3 \div 4$ times (although if we tested the contribution of blends to the analysed profiles, we ran the minimization routine several more times). Supposing that all analysed lines contain the signatures of the same magnetic field structure, we chose the initial locations in parameter space not far from the parameter set obtained from the strong line analysis.

In order to evaluate the fit errors (and therefore the uncertainties on the derived free parameters), we calculate deviations of the simulated profiles produced as a result of small variations of each of the free parameters, thus introducing a small shift along one axis in the $\chi^{2}$ hyper-space from the point of the function minimum value. Using this procedure, and taking into account the uncertainties of the observational data and the obtained minimal value of $\chi^{2}$-function, we can estimate the errors of the best-fit parameters.

\subsection{Results}

Our initial assumption that iron is uniformly distributed over the surface of 78 Vir appears to be valid, given that the analysed Fe II lines reveal no significant variability of the Stokes $I$ profiles with rotational phase. Each Fe II line (or group of lines) shown in Table 1 was analyzed independently of the others, using a stellar atmosphere model with $T_{\text {eff }}=9250 \mathrm{~K}, \log g=4.5$, $v_{\mathrm{t}}=0 \mathrm{~km} \mathrm{~s}^{-1}$. For each best-fit simulation the derived free model parameters are given in Table 3. During the simulation of some Fe II lines the Stokes $Q$ and $U$ profiles were not taken into account, and consequently the angle $\Omega$ is not determined for those lines.

The other data in Table 3 are derived from the free model parameters (Khalack et al. 2003). Here $\beta$ defines the angle between the magnetic dipole axis and the stellar rotation axis (the angle exists if these two axes cross each other). The distance of the magnetic dipole center from the center of the star and one-half of the magnetic dipole size are represented by variables $a_{0}$ and $a$, respectively. The variables $\left(B_{\mathrm{p}}, \lambda_{\mathrm{p}}, \delta_{\mathrm{p}}\right)$ and $\left(B_{\mathrm{n}}, \lambda_{\mathrm{n}}, \delta_{\mathrm{n}}\right)$ specify the location of the positive and negative magnetic poles at the stellar surface and the respective strength of the magnetic field.

\subsubsection{The strong lines}

The Fe II $\lambda 4923.927, \lambda 5018.44$ and $\lambda 5169.03$ lines are the most prominent Fe lines in the spectrum of $78 \mathrm{Vir}$, and show the clearest Stokes $Q$ and $U$ signatures. The first profile is formed essentially by the single line Fe II $\lambda 4923.927$ (see Table 2) and appears to contain no important blends. The agreement between the observed and simulated data for Fe II $\lambda 4923.927$ is similar to that shown in Fig. 2 (for Fe II 25018). Independent Stokes $I$ spectra (each corresponding to a slightly different phase) were obtained with each of the Stokes $V, Q$ and $U$ spectra, and although only one set of Stokes $I$ profiles are presented in the figure, all were taken into account during the calculation of the $\chi_{1}^{2}$-function (Eq. (3)). The best-fit values of the Stokes IVQU $\chi^{2}$-functions for the Fe II $\lambda 4923.927$ line are given in the third column of Table 3.

The second line profile is composed mainly of the Fe II $\lambda 5018.44 \AA$ line, but is also contaminated by contributions from the Ti I $\lambda 5017.95, \mathrm{Cr}$ I $\lambda 5018.15$ and Cr II $\lambda 5018.84$ lines. It seems that the Cr II $\lambda 5018.84 \AA$ is responsible for the blend in the red wing of the Stokes $I$ and $V$ profiles (Fig. 2). Supposing a uniform $\mathrm{Cr}$ distribution, this blend is well fit for a $\mathrm{Cr}$ abundance of $\log \mathrm{Cr} / N_{\text {tot }}=-3.38$ dex. The best fit $\chi^{2}$ values for the Stokes IVQU profiles of Fe II $\lambda 5018.44$ are given in the fourth column of Table 3.

In the case of Fe II $\lambda 5169.033$, the profile is blended by the weaker Fe I $\lambda 5168.898$ and $\lambda 5169.296$ lines (see Table 2). According to Kochukhov et al. (2004) $\log g f=-0.786$ given in the GRIFON list for the Fe I $\lambda 5169.296 \AA$ line is too high to match the solar spectrum, and they recommend to use a decreased $\log g f=-2.15$. This value is employed here for the respective profile simulation and provides much better agreement with the observed data. The behaviour of the best fit Stokes IVQU profiles with stellar rotational phase for this particular line is very similar to that shown in Fig. 2 for Fe II 25018.44 . The sixth column of Table 3 presents the best fit parameters and the model characteristics for Fe II $\lambda 5169.033$.

The concordance of the observed and simulated Stokes $I$ and $V$ profile variations as a function of stellar rotation is extremely good for these lines. On the other hand, the fit to the Stokes $Q$ and $U$ profiles is only approximate. The simulated profiles show generally the same intensity, qualitative structure and variability as the observations. However, even with these 

a) $I / I_{\mathrm{c}}$
b) $V / I_{\mathrm{c}}$
c) $Q / I_{\mathrm{c}}$
d) $U / I_{\mathrm{c}}$
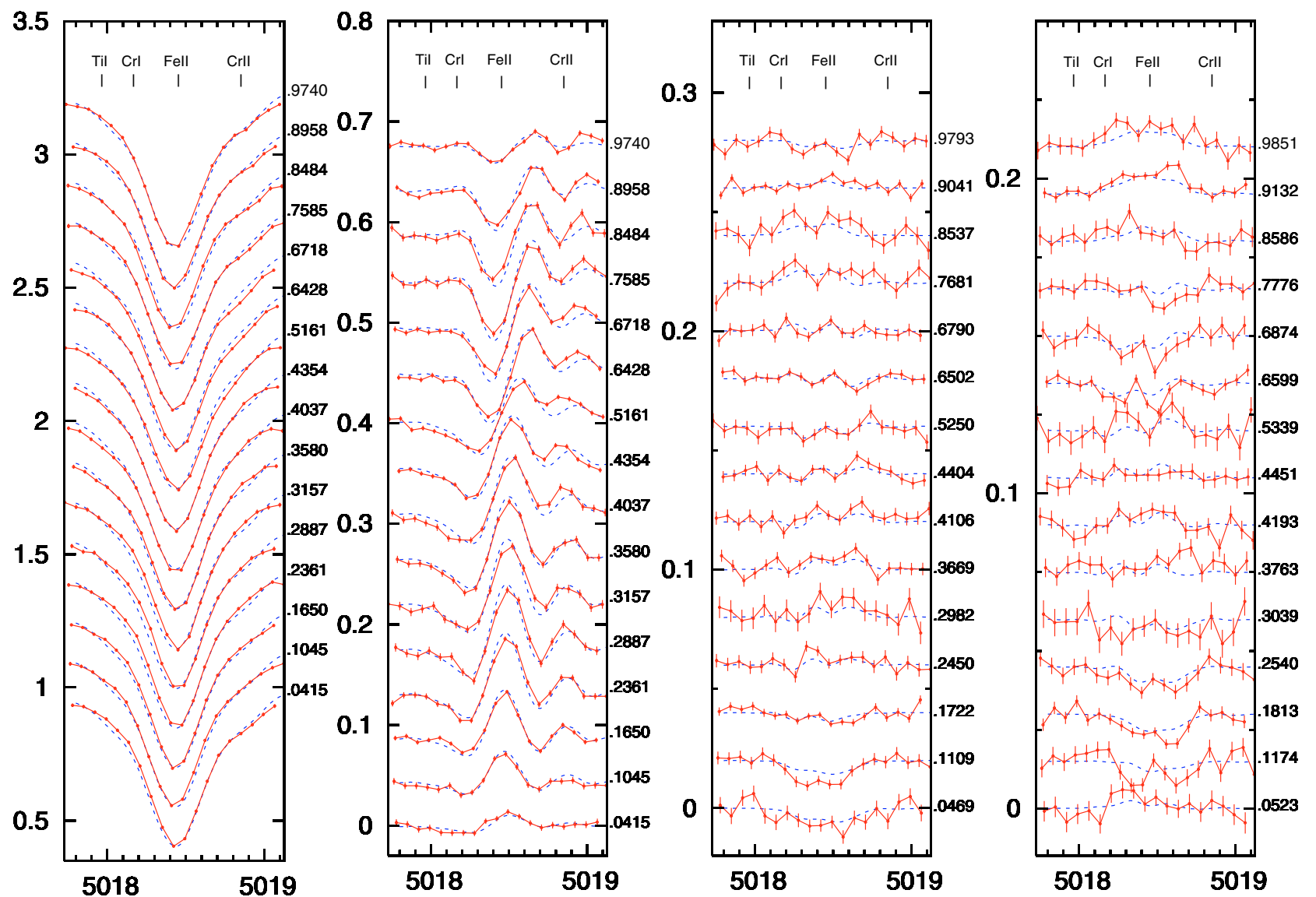

Fig. 2. Strong lines. Observed (solid lines) and simulated (dashed lines) Stokes $I$ a), $V$ b), $Q$ c) and $U$ d) line profiles for Fe II $\lambda 5018.44 \AA$ line. The vertical bars show the size of the respective observational errors. The observed and simulated profiles are shifted by $0.15,0.045,0.02$ and on 0.015 along the vertical axes for Stokes $I, V, Q$ and $U$ profiles respectively.

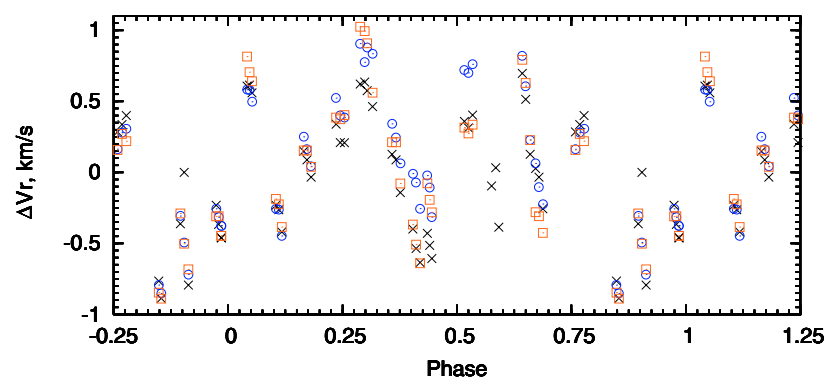

Fig. 3. The difference in radial velocity between the observed and calculated Stokes $I$ profiles for the Fe II lines $\lambda 4923.927 \AA$ (crosses), $\lambda 5018.44 \AA$ (open circles) and $\lambda 5169.033 \AA$ (open squares). The mean radial velocities $V_{\mathrm{r}}=-7.82 \mathrm{~km} \mathrm{~s}^{-1},-7.51 \mathrm{~km} \mathrm{~s}^{-1},-7.23 \mathrm{~km} \mathrm{~s}^{-1}$ obtained from the simulation of Fe II $\lambda 4923.927 \AA$, $\lambda 5018.44 \AA$, 15169.033 $\AA$ lines respectively (see Table 3), have been taken into account.

relatively noisy data, it is clear that the model does not reproduce the observations within the errors at some phases (for example, see phases $0.1174,0.5339$ and 0.9851 in Fig. 2).

In order to verify the assumption of a uniform surface distribution of iron in the atmosphere of $78 \mathrm{Vir}$, we have calculated the difference in radial velocity between the observed and simulated Stokes $I$ profiles for the strong Fe II lines $\lambda 4923.927, \lambda 5018.44$ and $\lambda 5169.033 \AA$ (see Fig. 3).

The mean (averaged for all the available observational phases) radial velocities obtained from the simulation are taken into account in the calculation of the velocities of the respective simulated profiles. Figure 3 shows that the derived differences in $V_{\mathrm{r}}$ almost coincide for all three lines. A moderate disagreement may exist only for the data obtained in the vicinity of rotational phase $\varphi=0.5$, when the negative magnetic pole is most visible. The variation of radial velocity, which shows a reasonably coherent variation with phase from $\mathrm{ab}-1$ to $+1 \mathrm{~km} \mathrm{~s}^{-1}$, may reflect a mildly non-uniform distribution of $\mathrm{Fe}$, unmodelled structure in the magnetic field, or other unaccounted-for physical processes in the stellar atmosphere.

\subsubsection{The moderate strength lines}

From the list of the Fe II lines selected for analysis (see Table 2) the $\lambda 4620.52, \lambda 5197.58, \lambda 6432.68$ and $\lambda 6516.08$ lines are weaker than the $\lambda 4923.927, \lambda 5018.44$ and $\lambda 5169.03$ lines. Due to the weaker polarised signal, a few spectra with 

a) $I / I_{\mathrm{c}}$
b) $V / I_{\mathrm{c}}$
c) $Q / I_{\mathrm{c}}$
d) $U / I_{\mathrm{c}}$
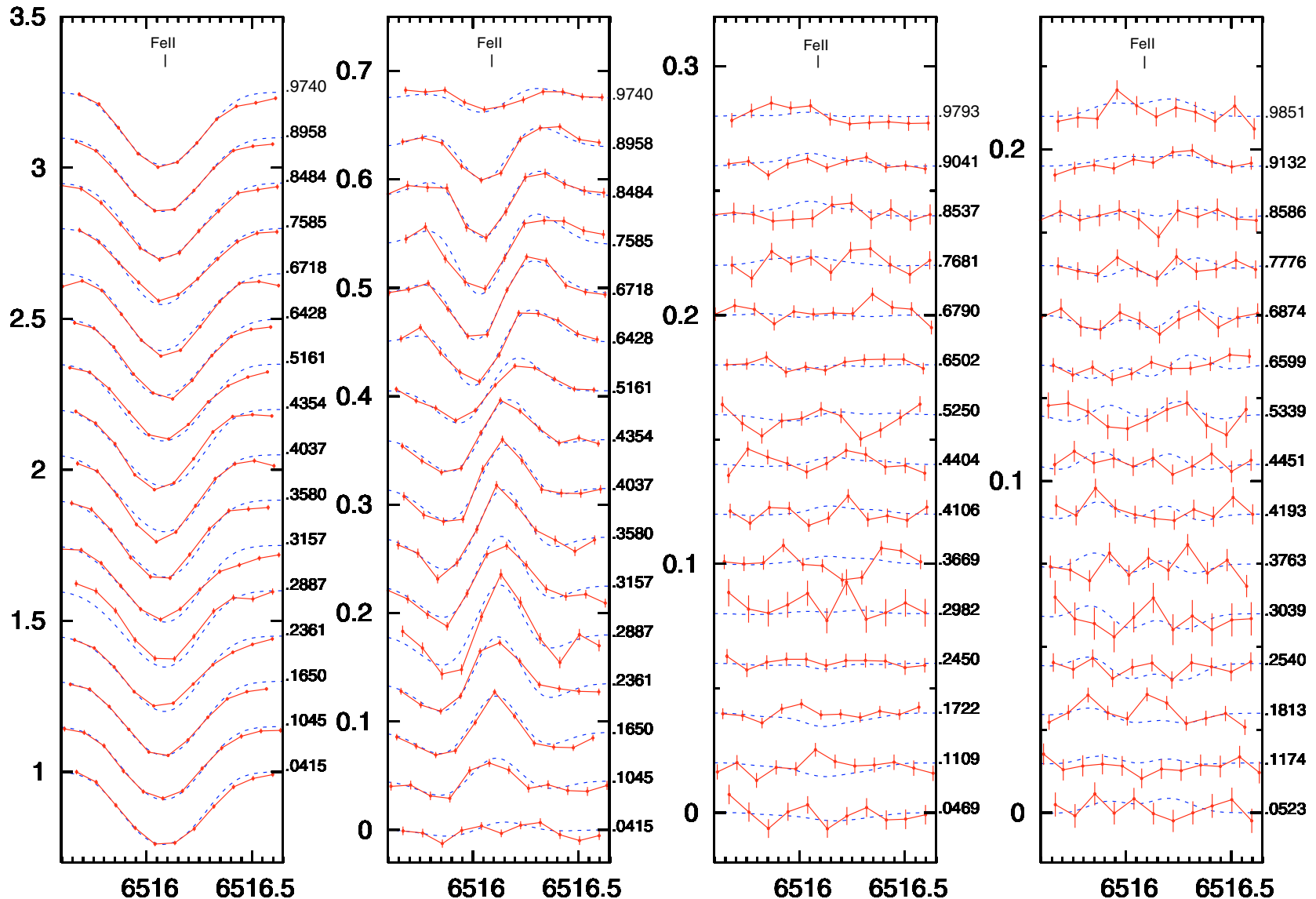

Fig. 4. The same as at the Fig. 2, but for Fe II $\lambda 6516.08 \AA$.

comparatively large observational errors have been excluded from the simulation (phases $0.5757,0.5847$ and 0.5914).

The line Fe II $\lambda 4620.521$ is primarily responsible for the formation of the observed profile. No blends were taken into account during the simulation in this case. The Fe II $\lambda 5197.577$ line is blended by the weak Fe II $\lambda 5197.48$ line, but provides the main contribution to the observed profile. The behaviour of the best fit Stokes IVQU profiles with stellar rotational phase for these lines is very similar to that shown in Fig. 4 for Fe II $\lambda 4620.521 \AA$. The first and seventh columns of Table 3 present the best fit parameters and the model characteristics for Fe II lines $\lambda 4620.521 \AA$ and $\lambda 5197.577 \AA$, respectively.

The Fe II $\lambda 6432.68 \AA$ line is also essentially unblended. The agreement between the observed and simulated Stokes IVQU data for this particular line is very similar to that presented by Fig. 4 . The Fe II $\lambda 6516.08 \AA$ line is the primary contributor to the formation of the corresponding observed Stokes IVQU profiles. The agreement of the best-fit simulation with the observed data for Fe II $\lambda 6516.08 \AA$ line is shown at Fig. 4. The tenth and eleventh columns at the Tables 3 contain the best fit parameters and model characteristics for the Fe II $\lambda 6432.68 \AA$ and $\lambda 6516.08 \AA$ lines, respectively. The best fit of the simulated Stokes IVQU profiles for the
Fe II $\lambda 6516.08 \AA$ line is statistically better than that obtained for Fe II $\lambda 6432.68 \AA$.

\subsubsection{Separate analysis of the Stokes I and $V$ spectra}

The other Fe II lines $\lambda 4635.316 \AA$, the $\lambda 5100 \AA$-group, $\lambda 5362.87 \AA$ and $\lambda 6247.56 \AA$ show no variability in the Stokes $Q$ and $U$ spectra and hence only the Stokes $I$ and $V$ spectra are taken into account during the simulation. These lines are selected for the analysis in order to check the resulting magnetic field structure of $78 \mathrm{Vir}$ on the basis of a more thorough line list.

The Fe II $\lambda 4635.316$ line is the main contributor to the formation of the corresponding observed Stokes $I$ and $V$ profiles, although it is blended by the comparatively weak Fe I $\lambda 4635.846 \AA$ line. The agreement between the observed and simulated data for this particular line is similar to that shown in Fig. 5. The superposition of the Fe II $\lambda 5100.607$, $\lambda 5100.664, \lambda 5100.727$ and $\lambda 5100.852$ lines is responsible for the formation of Stokes $I$ and $V$ profiles at $\sim 5100.7 \AA$ (see Table 2). Figure 5 shows the simulated Stokes $I$ and $V$ profiles, which fit well the observed profiles (see also the respective $\chi^{2}$ value in the fifth column of Table 3 ). 
a) $I / I_{\mathrm{c}}$

b) $V / I_{\mathrm{c}}$

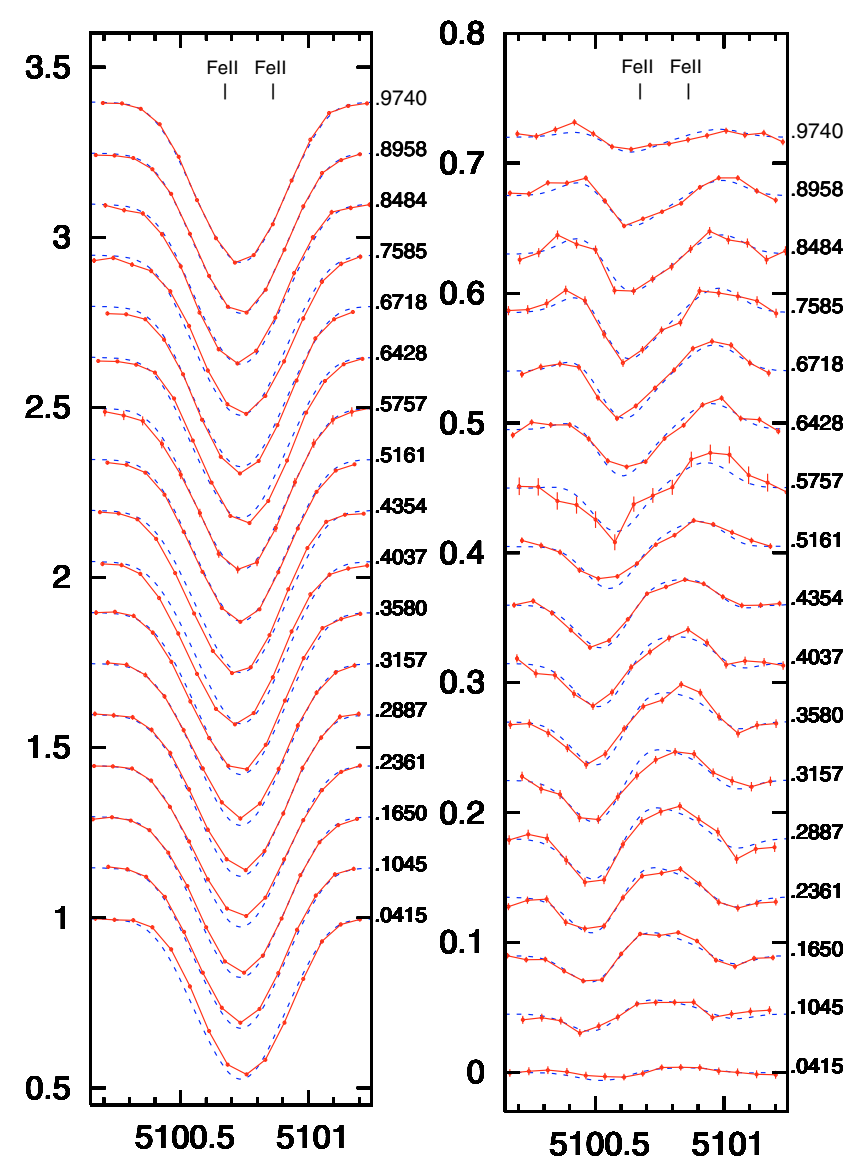

Fig. 5. Observed (solid lines) and simulated (dashed lines) Stokes $I$ a) and $V$ b) profiles for Fe II $\lambda 5100.607 \AA, \lambda 5100.664 \AA, \lambda 5100.727 \AA$ and $\lambda 5100.852 \AA$ lines. The vertical bars show the size of respective observational errors. The observed and simulated profiles are shifted on 0.15 , and on 0.045 along the vertical axes for Stokes $I$ and $V$ profiles correspondingly.

In the region of the Fe II $\lambda 5362.87$ line the observed Stokes $I$ and $V$ profiles are formed mainly by this line and by a weak contribution from the Fe II $\lambda 5362.74, \lambda 5362.98$, Cr I $\lambda 5362.87$ and $\mathrm{Cr}$ II $\lambda 5363.88$ lines. The Fe II $\lambda 6247.557$ line also provides the main contribution to the observed Stokes $I$ and $V$ profiles and is blended by the Fe II $\lambda 6247.35$ line. The best fit simulated data show almost the same fit quality as is shown in Fig. 5. Simulation of the Stokes $I$ and $V$ profiles in the regions of these lines results in almost the same configuration of the magnetic field structure as obtained from the analysis of the stronger Fe II lines, for which all four Stokes parameters were taken into account during the simulation.

\subsection{Integral magnetic field characteristics}

In order to check the agreement of the derived magnetic field model with other available magnetic field data for $78 \mathrm{Vir}$, we calculate the intensity-weighted, averaged (over the visible stellar disk) longitudinal magnetic field $B_{1}$ and the normalized equivalent widths of the Stokes $Q$ and $U$ profiles for all the

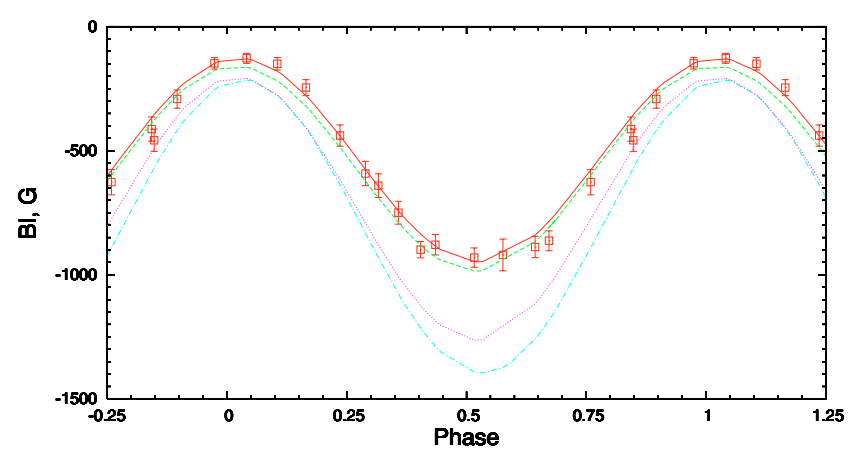

Fig. 6. Longitudinal magnetic field data with phase obtained from analysis of Fe II $\lambda 6432.68$ (solid line), Fe II $\lambda 5018.44$ (dashed line), Fe II $\lambda 6516.08$ (dotted line) and Fe II $\lambda 5197.58$ (dash-dotted line). The open squares with $1 \sigma$ error bars correspond to the LSD data published by Wade et al. (2000b).
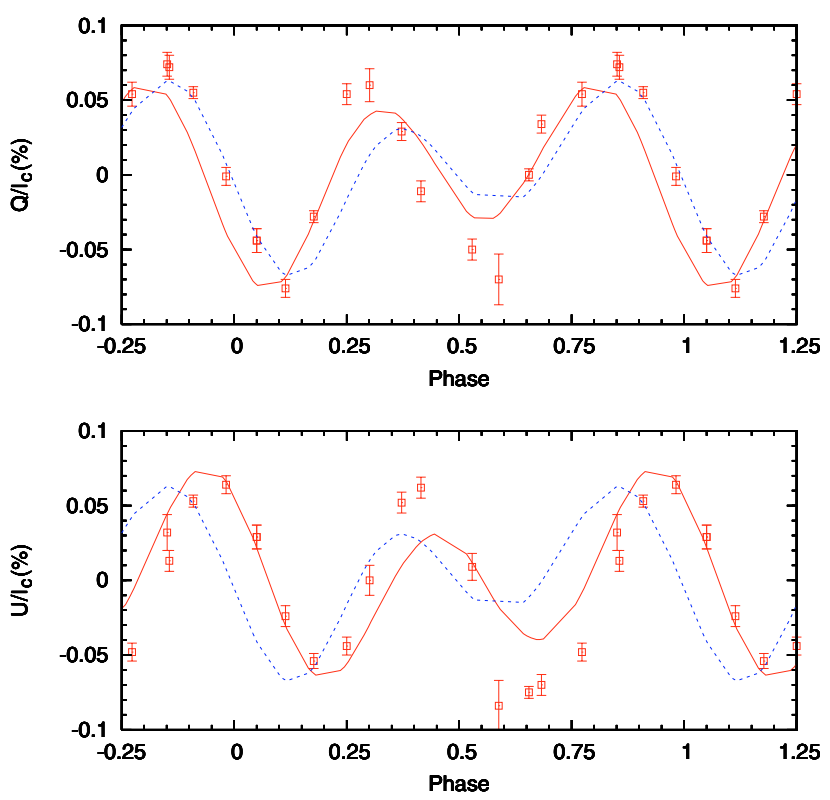

Fig. 7. Comparison of scaled net linear polarization data obtained from the best fit simulations of Fe II $\lambda 4923.927$ (solid line) and Fe II $\lambda 6516$ (dashed line), and from LSD profiles (Wade et al. 2000b) (open squares) for 78 Vir with respective $1 \sigma$ error bars.

analysed phases. The normalization procedure is performed in accordance with the method described by Wade et al. (2000b) over the passband of the analysed line profiles.

All the available longitudinal magnetic field measurements for 78 Vir are plotted in Fig. 6 of Leone \& Catanzaro (2001). This figure shows the good agreement of the $B_{1}$ measurements obtained by Wade et al. (2000b) using the Least-Squares Deconvolution (LSD) technique (Donati et al. 1997) with the majority of other observational data. Therefore, in this paper we just compare our results with the LSD longitudinal field data.

As demonstrated by Wade et al. (2000b), the 78 Vir Stokes $Q$ and $U$ LSD equivalent widths are proportional to the BBLP measured at that phase (Leroy 1995) with a line scaling factor. This factor might be different for the analysed lines (see the last column in Table 2), but is the same for the both Stokes $Q$ and $U$ equivalent widths for a particular spectral line. 

a) $I / I_{\mathrm{c}}$
b) $V / I_{\mathrm{c}}$
c) $Q / I_{\mathrm{c}}$
d) $U / I_{\mathrm{c}}$
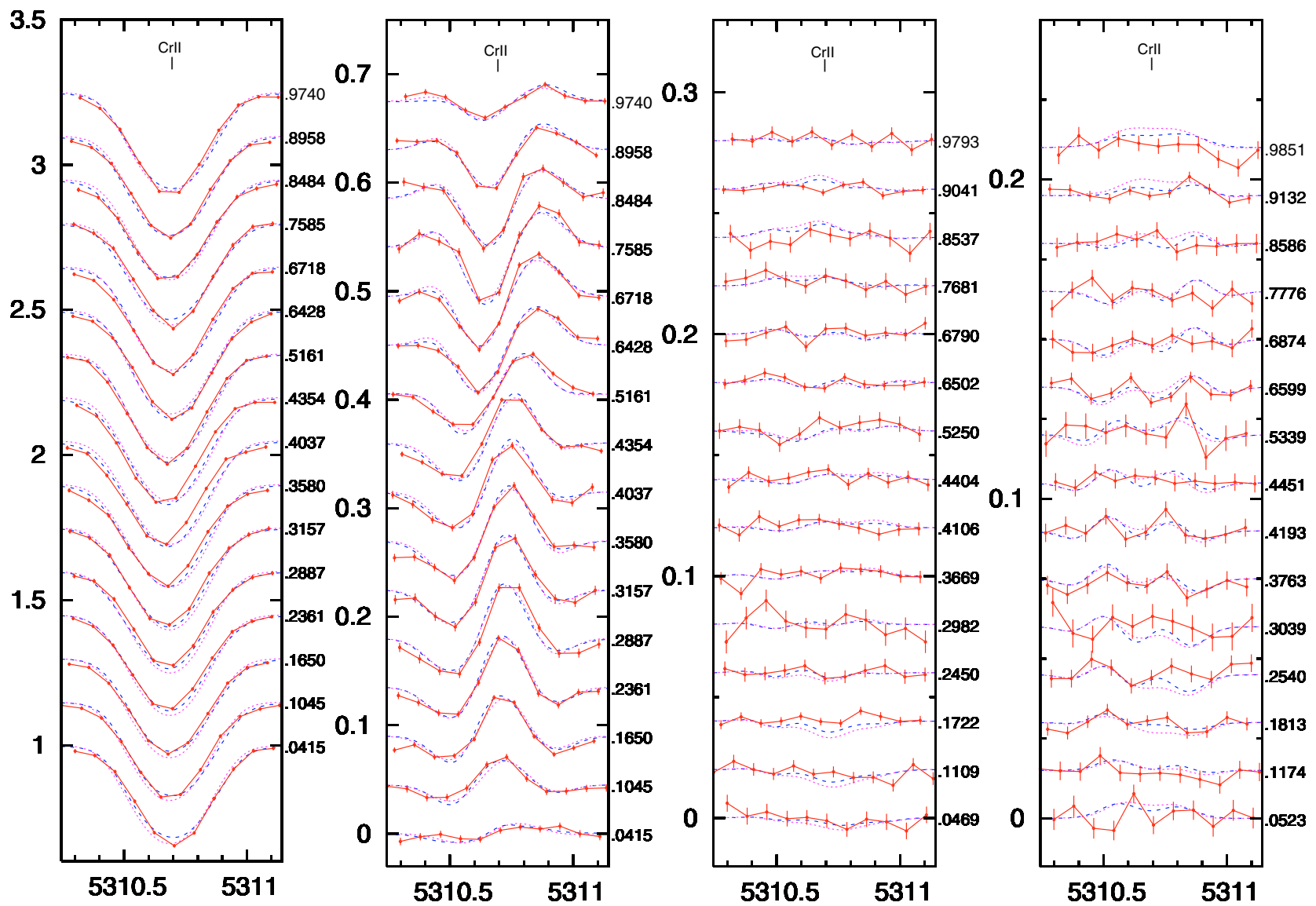

Fig. 8. The same as at the Fig. 2, but for Cr II $\lambda 5310.687 \AA$. Non-uniform chromium distribution in the stellar atmosphere is assumed during this simulation (dashed line). For comparison we show also the best fit simulation result $\left(\chi^{2}=4.24\right)$ for uniform chromium distribution (dotted line).

Neither the $B_{1}$ measurements nor the BBLP data are included directly into the model minimization procedure. However, given that the model successfully reproduces the Stokes profiles with which these quantities are fundamentally related, we should expect that the model magnetic field configuration is capable of reproducing them. Using the same stellar atmosphere model, the longitudinal field calculated from the model fits to the strongest Fe II line $\lambda 5018.44 \AA$ and to the weak Fe II line $\lambda 6432.68 \AA$ show a good agreement with the LSD $B_{1}$ data (see Fig. 6). The longitudinal fields calculated from the simulation results for the other Fe II lines appear to be shifted downward in longitudinal field intensity relative to the LSD $B_{1}$ variation (see Fig. 6).

The calculated Stokes $Q$ and $U$ equivalent widths for all the analysed line profiles are multiplied by the respective line scaling factor in order to fit them to the LSD Stokes $Q$ and $U$ data. For each analysed line a scaling factor is determined with the help of the Least Squares method. The respective results are given in Table 2, taking into account that the LSD equivalent widths of Stokes $Q$ and $U$ profiles have a line scaling factor of 0.1 (Wade et al. 2000b) from comparison with the BBLP data (Leroy 1995). Figure 7 presents the Stokes $Q$ and $U$ equivalent widths variability with phase, derived from the best fit simulations of Fe II $\lambda 4923.927$ (with $\Omega=109^{\circ}$ ) and Fe II $\lambda 6516.08$ (with $\Omega=128^{\circ}$ ). They are scaled by a factor of 0.08 (for 24923 ) and 0.03 (for 26516 ) and plotted over the Stokes $Q$ and $U$ equivalent widths derived from LSD profiles. It is remarkable that the simulated polarimetric data for Fe II $\lambda 4923.927 \AA$ and $\lambda 5018.44 \AA$ lines have almost the same scaling factor as the LSD Stokes $Q$ and $U$ equivalent widths. Theoretically, the line scaling factor depends on the Landé factor and on the degree of line saturation (Wade et al. 2000a). Table 2 shows that in general the lines with a high mean Landé factor have also a comparatively high line scaling factor. Nevertheless, this dependence is not clearly pronounced by the sample of derived data. This is because, for some lines, this effect is reduced by blend contamination of the analysed Stokes $Q$ and $U$ profiles (for example the Fe II $\lambda 5100$ line group, $\lambda 5197.03$ and $\lambda 5362.87$ lines) or by weak Stokes $Q$ and $U$ profile variability, which results in a comparatively low precision of the calculated equivalent widths.

The LSD net linear polarisation (in the same way as the BBLP) reflect the contributions of many spectral lines with significant polarization. These lines belong to a number of chemical elements, which apparently have non-uniform and usually quite different abundance distributions. Such a composition of 
Table 4. The same as at Table 3, but for Cr II and Ti II lines.

\begin{tabular}{|c|c|c|c|c|c|c|c|c|c|c|}
\hline Line & Cr II & Cr II & Cr II & Cr II & Cr II & Cr II & & Ti II & Ti II & \\
\hline$\AA$ & 4592 & 4634 & 5237 & 5310 & 5407 & 5421 & $\sigma_{\mathrm{er}}$ & 5188 & 5336 & $\sigma_{\mathrm{er}}$ \\
\hline$\chi_{I}^{2}$ & 14.82 & 13.12 & 10.51 & 6.47 & 6.20 & 9.48 & & 21.53 & 12.45 & \\
\hline $4 \chi_{V}^{2}$ & 8.83 & 6.39 & 12.70 & 8.64 & 11.11 & 11.50 & & 24.28 & 9.30 & \\
\hline $6 x_{Q}^{2}$ & 5.62 & 6.18 & - & 8.18 & 8.55 & 11.76 & & 8.77 & 5.79 & \\
\hline $6 x_{U}^{2}$ & 6.48 & 5.88 & - & 6.63 & 9.17 & 12.33 & & 7.45 & 6.45 & \\
\hline$\chi_{\mathrm{w}}^{2}$ & 8.95 & 7.89 & 11.60 & 7.49 & 8.76 & 11.27 & & 15.51 & 8.50 & \\
\hline$\chi^{2}$ & 4.76 & 4.18 & 6.84 & 2.77 & 2.98 & 4.09 & & 7.58 & 4.20 & \\
\hline Qr, kG & 175 & 186 & 185 & 182 & 161 & 171 & 30 & 287 & 271 & 40 \\
\hline$a_{1}, 10^{-3}$ & 10.2 & 9.5 & 9.7 & 8.9 & 9.6 & 8.6 & 1.5 & 3.7 & 3.8 & 1.4 \\
\hline$\lambda_{1}$ & $19^{\circ}$ & $15^{\circ}$ & $16^{\circ}$ & $25^{\circ}$ & $17^{\circ}$ & $21^{\circ}$ & $5^{\circ}$ & $28^{\circ}$ & $31^{\circ}$ & $17^{\circ}$ \\
\hline$\delta_{1}$ & $-41^{\circ}$ & $-42^{\circ}$ & $-45^{\circ}$ & $-49^{\circ}$ & $-40^{\circ}$ & $-46^{\circ}$ & $5^{\circ}$ & $-39^{\circ}$ & $-41^{\circ}$ & $19^{\circ}$ \\
\hline$a_{2}, 10^{-3}$ & 4.4 & 4.1 & 3.8 & 3.9 & 3.6 & 3.7 & 0.3 & 2.8 & 3.5 & 1.0 \\
\hline$\lambda_{2}$ & $103^{\circ}$ & $120^{\circ}$ & $111^{\circ}$ & $115^{\circ}$ & $109^{\circ}$ & $120^{\circ}$ & $8^{\circ}$ & $113^{\circ}$ & $113^{\circ}$ & $19^{\circ}$ \\
\hline$\delta_{2}$ & $-17^{\circ}$ & $-18^{\circ}$ & $-17^{\circ}$ & $-12^{\circ}$ & $-2^{\circ}$ & $-19^{\circ}$ & $10^{\circ}$ & $-8^{\circ}$ & $-14^{\circ}$ & $12^{\circ}$ \\
\hline$\Omega$ & $109^{\circ}$ & $117^{\circ}$ & - & $123^{\circ}$ & $115^{\circ}$ & $109^{\circ}$ & $14^{\circ}$ & $97^{\circ}$ & $90^{\circ}$ & $19^{\circ}$ \\
\hline$i$ & $26^{\circ}$ & $26^{\circ}$ & $26^{\circ}$ & $26^{\circ}$ & $22^{\circ}$ & $27^{\circ}$ & $5^{\circ}$ & $25^{\circ}$ & $18^{\circ}$ & $5^{\circ}$ \\
\hline $\log \left(E l / N_{\text {tot }}\right)$ & -3.90 & -4.02 & -4.04 & -3.91 & -4.18 & -4.17 & 0.19 & -5.48 & -5.68 & 0.22 \\
\hline$V_{\mathrm{r}}$ & -8.25 & -8.02 & -8.80 & -8.41 & -8.05 & -8.25 & 1.23 & -5.61 & -7.91 & 1.26 \\
\hline$V_{\mathrm{e}} \sin i$ & 11.7 & 10.8 & 11.8 & 11.5 & 10.9 & 12.2 & 1.1 & 13.4 & 13.3 & 1.5 \\
\hline$\beta$ & $123^{\circ}$ & $119^{\circ}$ & $126^{\circ}$ & $131^{\circ}$ & $127^{\circ}$ & $124^{\circ}$ & $5^{\circ}$ & $117^{\circ}$ & $112^{\circ}$ & $5^{\circ}$ \\
\hline$a_{0}, 10^{-3}$ & 6.1 & 5.2 & 5.5 & 5.2 & 5.1 & 4.9 & 1.4 & 2.5 & 2.9 & 1.7 \\
\hline$a, 10^{-3}$ & 5.0 & 5.1 & 5.0 & 4.6 & 5.1 & 4.4 & 1.4 & 2.1 & 2.2 & 1.7 \\
\hline$B_{\mathrm{p}}, \mathrm{kG}$ & 3.52 & 3.86 & 3.71 & 3.36 & 3.32 & 3.05 & 0.4 & 2.46 & 2.41 & 0.5 \\
\hline$\lambda_{\mathrm{p}}$ & $-11^{\circ}$ & $-10^{\circ}$ & $-11^{\circ}$ & $-8^{\circ}$ & $-8^{\circ}$ & $-7^{\circ}$ & $8^{\circ}$ & $-18^{\circ}$ & $-23^{\circ}$ & $8^{\circ}$ \\
\hline$\delta_{\mathrm{p}}$ & $-33^{\circ}$ & $-29^{\circ}$ & $-36^{\circ}$ & $-41^{\circ}$ & $-37^{\circ}$ & $-34^{\circ}$ & $10^{\circ}$ & $-27^{\circ}$ & $-22^{\circ}$ & $11^{\circ}$ \\
\hline$B_{\mathrm{n}}, \mathrm{kG}$ & -3.44 & -3.78 & -3.62 & -3.29 & -3.24 & -2.99 & 0.4 & -2.45 & -2.41 & 0.5 \\
\hline$\lambda_{\mathrm{n}}$ & $169^{\circ}$ & $170^{\circ}$ & $169^{\circ}$ & $172^{\circ}$ & $171^{\circ}$ & $173^{\circ}$ & $8^{\circ}$ & $161^{\circ}$ & $156^{\circ}$ & $8^{\circ}$ \\
\hline$\delta_{\mathrm{n}}$ & $33^{\circ}$ & $29^{\circ}$ & $35^{\circ}$ & $40^{\circ}$ & $37^{\circ}$ & $34^{\circ}$ & $10^{\circ}$ & $27^{\circ}$ & $22^{\circ}$ & $11^{\circ}$ \\
\hline
\end{tabular}

polarised features results in LSD (or BBLP) data that may differ substantially from the Stokes $Q$ and $U$ equivalent widths derived for a particular Fe II line (see Fig. 7). This is illustrated by the rather large differences between the two simulated curves in Fig. 7. Moreover, lines of elements with non-uniform abundance distributions can provide substantially different values of the sky-projected position angle of the stellar rotation axis (see Sect. 4.4), and can exhibit totally different net linear polarisation variations (e.g. $180^{\circ}$ out of phase with those shown here). The spectral resolution and the observational errors of the available spectra do not allow us to map confidently the detailed distribution of chemical abundances nor the local patterns of the surface magnetic field. The uncertainties in the details of the abundance distributions and the magnetic field structure limit the precision of $\Omega$ as well as the quality of simulated Stokes $Q$ and $U$ equivalent widths. Given these limitations, we would characterise the agreement of the observed and simulated variations shown in Fig. 7 as very acceptable.

\subsection{Lines of other elements}

78 Vir is a chromium-rich star (Cowley et al. 1969) and has some strong CrII lines, which are located in the observed spectral range. The most prominent of them, Cr II $\lambda 4592.049, \lambda 4634.07, \lambda 5237.329, \lambda 5310.687, \lambda 5407.604$ and $\lambda 5420.922 \AA$, have been selected for simulation.
Atomic parameters of spectral lines were extracted from the VALD database (Kupka et al. 1999) and from Raassen \& Uylings (1998) (ftp://ftp.wins.uva.nl/pub/orth) in the case of $\mathrm{Cr}$ II lines. These lines are not significantly blended by lines of other elements and show variability of the Stokes $I V$ parameters with phase. Some show marginallydetected Stokes $Q U$ signatures as well.

We analyse also the two strong TiII $\lambda 5188.68$ and $\lambda 5336.771$ lines. These Ti lines are not significantly blended (although we take into account a small contribution of the V I $\lambda 5188.885$ blend to the profile formed by Ti II $\lambda 5188.68)$.

Table 4 presents the results of the best fit simulations for the $\mathrm{Cr}$ and $\mathrm{Ti}$ lines. The best fit quality is on average similar to that obtained for the Fe II lines. The Stokes I profiles for these particular lines vary weakly with rotational phase, suggesting the presence of non-uniform distributions of Cr II and $\mathrm{Ti}$ II in the atmosphere of 78 Vir. To take into account the non-uniform abundance distributions the stellar surface is divided into 30 areas, each characterised by an independent local abundance of the analysed chemical element. The local abundances are included as free parameters in the simulation procedure and the model operates in this case with 41 parameters. The best fit simulations of the aforementioned lines tentatively suggest that chromium is enhanced in the vicinity of the negative magnetic pole, while titanium is underabundant in this region (see Fig. 10). The improvement in the fit to Cr II $\lambda 5310$ 

a) $I / I_{\mathrm{c}}$
b) $V / I_{\mathrm{c}}$
c) $Q / I_{\mathrm{c}}$
d) $U / I_{\mathrm{c}}$
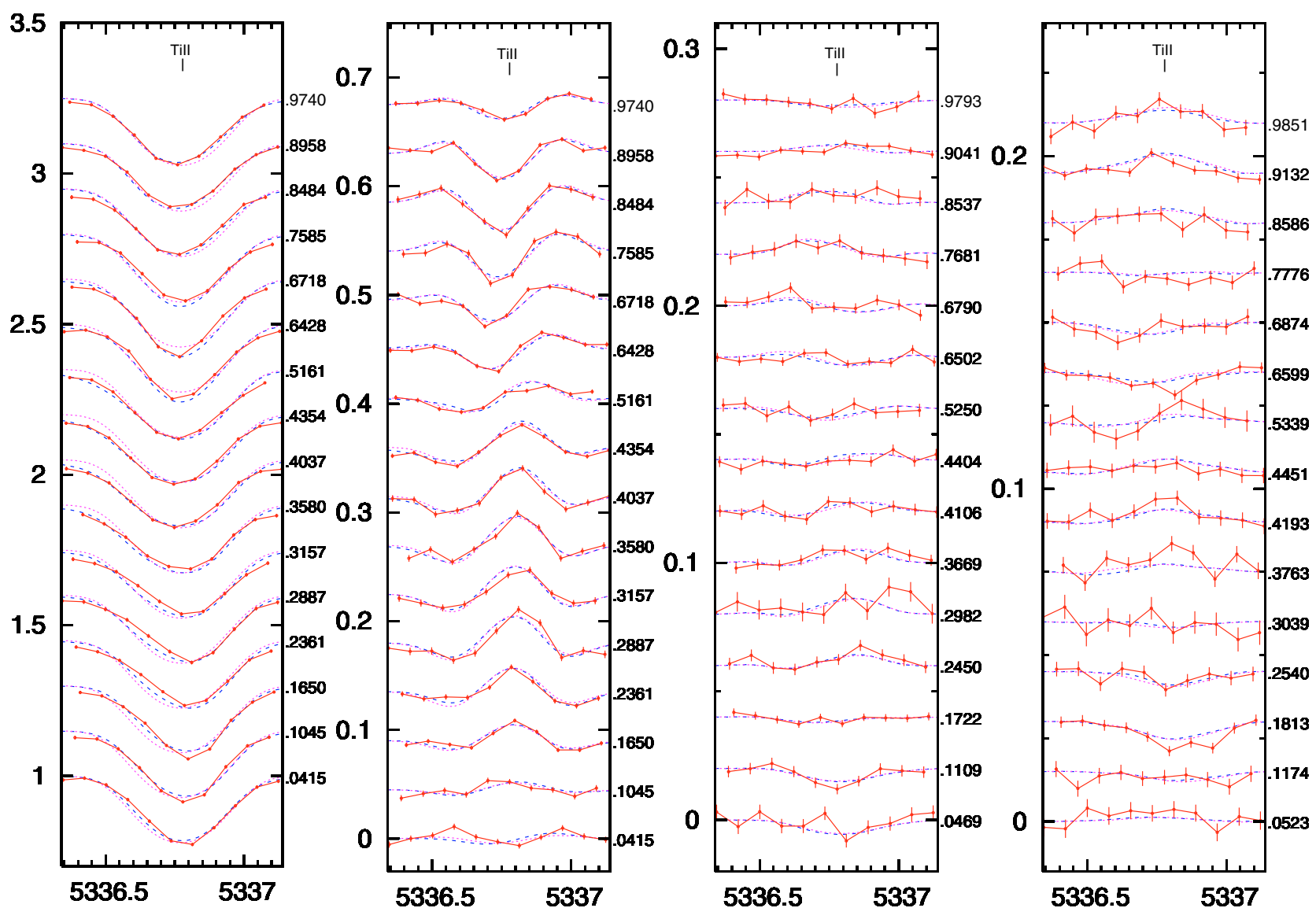

Fig. 9. The same as at the Fig. 2, but for Ti II $\lambda 5336.771 \AA$ A. Non-uniform titanium distribution in the stellar atmosphere is assumed during this simulation (dashed line). For comparison we show also the best fit simulation result $\left(\chi^{2}=4.58\right)$ for a uniform titanium distribution (dotted line).

a)
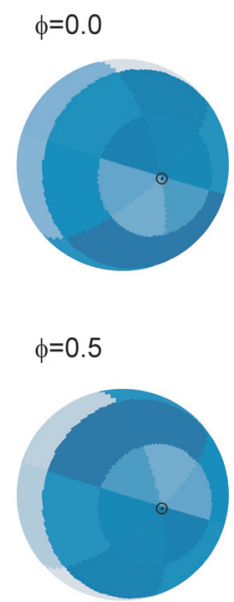

$\phi=0.25$

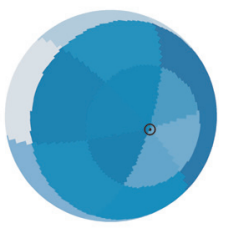

$\phi=0.75$

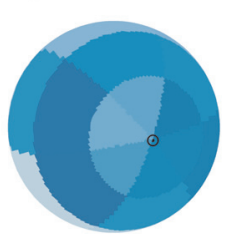

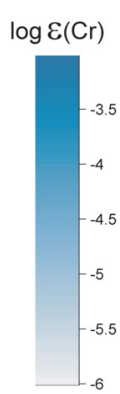

$\phi=0.0$

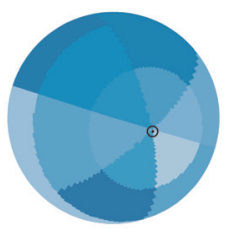

$\phi=0.5$

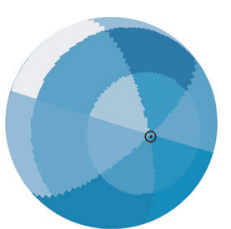

b)

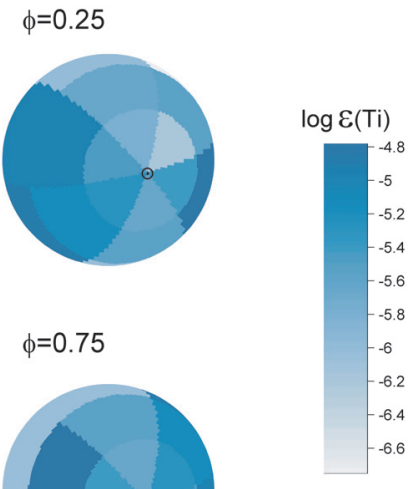

Fig. 10. Illustration of the a) chromium and b) titanium abundance distribution in the stellar atmosphere of 78 Vir. Location of the positive rotation pole is specified by open circle. The stellar image has been rotated in the plane of the page by the positional angle $\Omega=110^{\circ}$. 
resulting from a non-uniform abundance distribution is highly significant (reduction of $\chi_{I}^{2}$ by over $40 \%$ ), although the improvement for Ti II $\lambda 5336$ is much less so (just 10\%). The averaged chromium and titanium abundances are given in Table 4.

It is remarkable that the best fit simulations of $\mathrm{Cr}$ II lines provide almost the same global magnetic field structure and the sky-projected position angle of the stellar rotation axis as the Fe lines (see Tables 3, 4). Meanwhile, the Ti II simulation results in somewhat lower values of $\Omega$ and $\beta$. These differences may results from the non-uniform Ti II distribution in the stellar atmosphere.

\section{Discussion}

The abundance of Fe II derived in this study is $\log \mathrm{Fe} / N_{\text {tot }}=$ $-3.16 \pm 0.20$ (see Table 3). From analysis of spectra of $78 \mathrm{Vir}$ in the regions $3850-3870 \AA$ and $3868-4650 \AA$ Adelman (1973b) obtained an Fe II abundance of $\log \mathrm{Fe} / N_{\mathrm{H}}=$ -2.89 dex. The differences in the iron abundances may be due to the inclusion/exclusion of polarized transfer, microturbulence, effects of Balmer line wings, etc., as well as from the different effective temperatures that have been employed for the abundance analysis (for 78 Vir Adelman (1973a) applied $T_{\text {eff }}=9950 \mathrm{~K}$, significantly higher than the temperatures employed in our model.

For the 11 different $\mathrm{Fe}$ features studied here, we observe no systematic difference in strength between weak and strong lines. Therefore, the results of this study are consistent with the absence of important $\mathrm{Fe}$ stratification in the atmosphere of 78 Vir.

According to our results the global magnetic field structure of 78 Vir is well-described by a slightly decentered magnetic dipole. Usually the starting point of a simulation (in the free parameter hyperspace) is chosen to correspond to a central magnetic dipole, but for all the analysed lines the final model results in a slightly decentered magnetic dipole. This fact is in good qualitative agreement with the results previously obtained by Borra (1980). In that paper Borra considered the classical decentered dipole model, where the magnetic dipole size is insignificant in comparison with the stellar radius, and obtained $a_{0}=0.2$. Our model provides a smaller $a_{0}=0.006 \pm 0.002$ due to the non-zero dipole size $(2 a=0.008 \pm 0.003)$, that allows us to take into account the non-symmetrical (with respect to the stellar center) magnetic field configuration of the star. The magnetic field intensity and location of the positive and negative magnetic poles in the stellar rotational reference frame are:

$B_{\mathrm{p}}=3.4 \pm 0.7 \mathrm{kG}, \quad \lambda_{\mathrm{p}}=-9^{\circ} \pm 5^{\circ}, \quad \delta_{\mathrm{p}}=-33^{\circ} \pm 12^{\circ} ;$

$B_{\mathrm{n}}=-3.3 \pm 0.7 \mathrm{kG}, \quad \lambda_{\mathrm{n}}=171^{\circ} \pm 5^{\circ}, \quad \delta_{\mathrm{n}}=33^{\circ} \pm 12^{\circ}$.

Due to the shift of the dipole from the stellar centre, the positive magnetic pole has a slightly stronger field intensity than the negative pole, but not all the analysed lines are equally sensitive to this difference. Nevertheless, the final model is hardly distinguishable from a symmetric central dipole model, which provides a slightly poorer agreement between the simulated and observed data. For Fe II lines the centered dipole model results in a $\chi^{2}$-function which exceeds by $3 \% \div 5 \%$ the best fit result
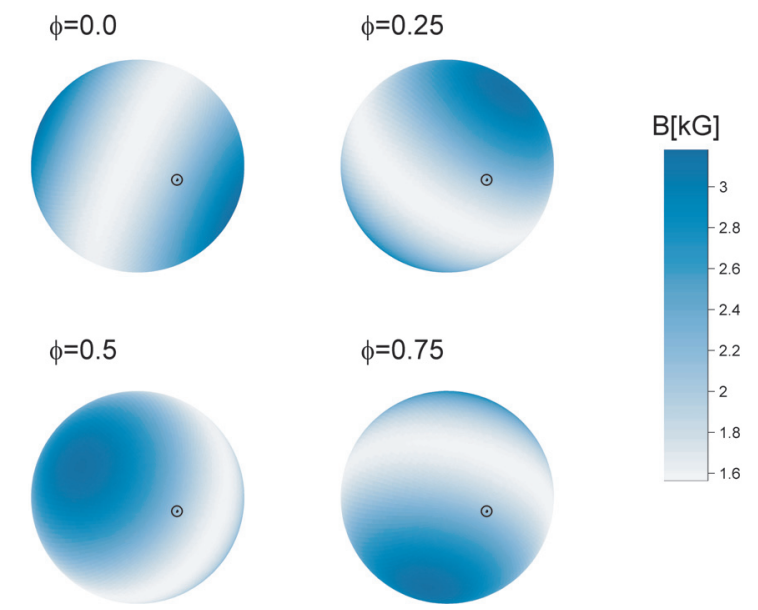

Fig. 11. Illustration of the surface magnetic field geometry of 78 Vir. The location of the positive rotation pole is indicated by the open circle. We always see the negative magnetic pole and only for phase $\varphi=0.0$ are the positive and negative magnetic poles visible simultaneously. The stellar image has been rotated in the plane of the page by the positional angle $\Omega=110^{\circ}$.

from the decentered dipole model. This fact can be related to the quality of the data and to the model sensitivity. The dipole offset could be a result of the particular geometry of $78 \mathrm{Vir}$, because we never completely see the positive magnetic pole of the star. The modeled global magnetic field configuration of 78 Vir is illustrated in Fig. 11 according to the best fit results obtained for Fe II $\lambda 5018 \AA$. Five of the Fe II lines analysed using the entire Stokes vector result in a plane-of-sky orientation of the rotational axis of $\Omega=110^{\circ} \pm 17^{\circ}$, while the last two lines (Fe II $\lambda 6432 \AA$ and $\lambda 6516 \AA$ ), with comparatively weaker variability of the observed Stokes $Q$ and $U$ profiles, provide higher values of this angle. Figure 12 shows the dependence of the $\chi_{Q}^{2}$ and $\chi_{U}^{2}$ functions on the angle $\Omega$ for the three strong Fe II lines (see Sect. 4.2.1). Both functions reach their minima at $\Omega=109^{\circ}$ and $\Omega=289^{\circ}$. The second value describes a model which is indistinguishable from the configuration specified in Table 3 due to the definition of the Stokes $Q$ and $U$ parameters.

As discussed in Sect. 3, 78 Vir is a probable member of the Ursa Major stream. It approaches us with a mean radial velocity $V_{\mathrm{r}}=-8.1 \pm 1.0 \mathrm{~km} \mathrm{~s}^{-1}$ (Wade et al. 2000a). This velocity varies by about $\pm 2 \mathrm{~km} \mathrm{~s}^{-1}$ with the phase of stellar rotation (Preston 1969, this work). The possible variability of the radial velocity is not taken into account in the simulation procedure. Respectively, our estimations of the radial velocity for different $\mathrm{Fe}$ II lines are distributed around the aforementioned value within the range $\pm 1.5 \mathrm{~km} \mathrm{~s}^{-1}$.

Nevertheless, the $V_{\mathrm{r}}$ variability through the rotational cycle has been determined from the strong Fe II lines $\lambda 4923.927 \AA$ $\lambda 5018.44 \AA$ and $\lambda 5169.033 \AA$ (see Sect. 4.2.1) and for the other $\mathrm{Fe}$ II lines as well. We show that the mean radial velocity derived from the simulation varies from line to line (see Table 3 ). The other Fe II lines provide a similar $\Delta V_{\mathrm{r}}$ variability. The precision of the mean radial velocity determination depends on the observational errors and on the quality of the description of the 
a)

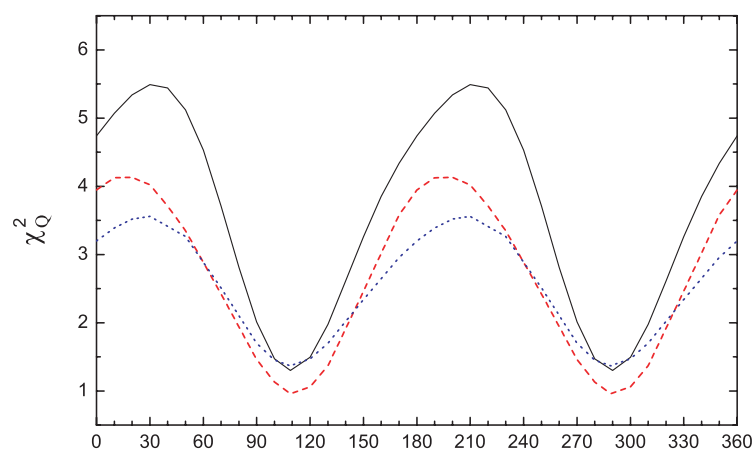

$\Omega$ b)

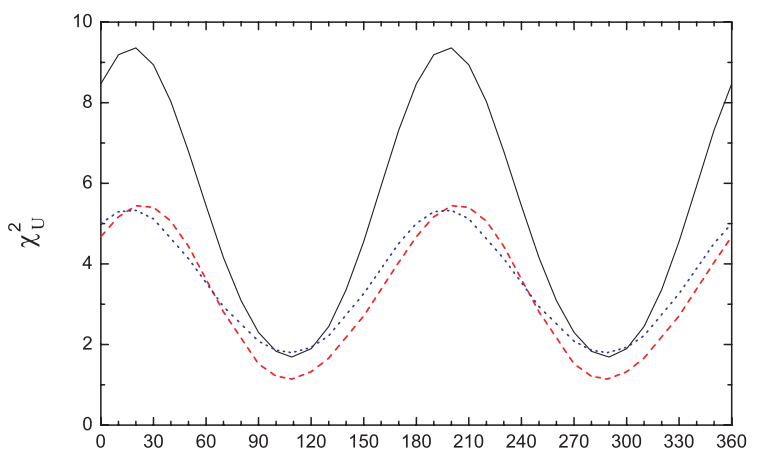

$\Omega$

Fig. 12. Dependence of a) $\chi_{Q}^{2}$ and b) $\chi_{U}^{2}$ on the angle $\Omega$ calculated for three strong lines Fe II $\lambda 4923.927 \AA$ (solid line), $\lambda 5018.44 \AA$ (dashed line) and $\lambda 5169.03 \AA$ (dotted line). The calculations were performed under the condition of stability of all the best fit parameters for these lines (see Table 3 ) except the $\Omega$.

surface magnetic field structure. For the blended profiles it also depends on the accuracy of oscillator strengths.

The analysed lines show weak, coherent variations of $\Delta V_{\mathrm{r}}$ with the period of stellar rotation (see Fig. 3). This suggests a possible moderately non-uniform distribution of Fe II. Nevertheless, the non-uniform iron distribution will not lead to a significantly different surface magnetic field structure. The simulation of Cr II lines with the assumption of a non-uniform chromium distribution resulted in almost the same field structure that we obtained from the simulation of Fe II lines.

The other best fit parameters are the rotational axis inclination $i=24^{\circ} \pm 5^{\circ}$ and the dipole axis obliquity $\beta=124^{\circ} \pm$ $5^{\circ}$, which are in a good agreement with the estimates of Leroy et al. (1996). The derived $V_{\mathrm{e}} \sin i=12 \pm 1 \mathrm{~km} \mathrm{~s}^{-1}$ provides $V_{\mathrm{e}}=29 \pm 4 \mathrm{~km} \mathrm{~s}^{-1}$ and is consistent with that of Preston (1971).

The derived surface magnetic field variability interval ranges from $2.1 \mathrm{kG}$ to $3.2 \mathrm{kG}$ and covers the value $B_{\mathrm{s}}=$ $2.9 \mathrm{kG}$ estimated by Preston (1971). Besides, as Fig. 6 shows, the longitudinal magnetic field variation obtained from the two Fe II lines simulation is also in good agreement with the LSD $B_{1}$ data (Wade et al. 2000b). These facts justify the applicability of the slightly decentered magnetic dipole model with the aforementioned values of free parameters for the global magnetic field structure description at 78 Vir.

Unfortunately in the case of 78 Vir the intensity of the Stokes $Q$ and $U$ profiles is similar to the observational errors. The data are unsuitable for performing an analysis of the small-scale structure of the magnetic field, using a more sophisticated technique such as Magnetic Doppler Imaging (MDI; Kochukhov \& Piskunov 2002). However, it appears, given the relatively good agreement between the intensity, structure and variability of the observed and simulated Stokes $Q U$ profiles, that the real magnetic field of 78 Vir does not depart strongly from the configuration derived here. At the same time, we have noted that the Stokes $Q U$ profiles are not, at some phases, fit to within their errors. Therefore these data, notwithstanding their relatively low $\mathrm{S} / \mathrm{N}$, already suggest the limitations of the MCD model framework.
A similar analysis of the Stokes $I V$ profile variability was performed by Kochukhov et al. (2002) for $\alpha^{2} \mathrm{CVn}$. Those authors applied MDI using "multipolar regularization", analysing the Stokes $I V$ line profiles and obtained a good agreement between observed and computed profiles for Fe II, Cr II and Si II lines. The quality of the Stokes $I$ and $V$ profile fits obtained in this paper is only marginally poorer than that obtained by Kochukhov et al. (2002), although remarkably the magnetic field model framework employed here is much simpler.

New spectro-polarimetric observations of 78 Vir with higher spectral resolution and signal-to-noise ratio (especially for the Stokes $Q$ and $U$ spectra) are required for further refinement of the global magnetic field structure. With such data, a model with additional parameters (such as MDI) could be applied, in order to study the local magnetic field topology and the detailed relationship between the magnetic field and the abundance distributions.

Acknowledgements. The authors are grateful to Prof. John Landstreet and to the referee, Dr. O. Kochukhov, for their valuable remarks and advice that have led to the improvement of this paper. The authors acknowledge grant support from the Natural Sciences and Engineering Research Council of Canada, and the Department of National Defence of Canada (DND-ARP).

\section{References}

Adelman, S. J. 1973a, ApJ, 183, 95

Adelman, S. J. 1973b, ApJS, 26, 1

Babcock, H. W. 1947, ApJ, 105, 105

Baudrand, J., \& Böhm, T. 1992, A\&A, 259, 711

Bessell, M. S., Castelli, F., \& Plez, B. 1998, A\&A, 333, 231

Borra, E. F. 1980, ApJ, 235, 915

Borra, E. F., \& Landstreet, J. D. 1980, ApJS, 42, 421

Catalano, F. A., \& Leone, F. 1994, A\&AS, 108, 595

Cowley, A., Cowley, C., Jaschek, M., \& Jaschek, C. 1969, AJ, 74, 375

Cayrel de Strobel, G., Soubiran, C., Friel, E. D., et al. 1997, A\&AS, 124, 299

Donati, J.-F., Catala, C., Wade, G. A., et al. 1999, A\&AS, 134, 149

Donati, J.-F., Semel, M., Carter, B. D., et al. 1997, MNRAS, 291, 658

ESA 1997, The Hipparcos and Tycho Catalogues (ESA SP-1200; Noordwijk: ESA) 
Flower, P. J. 1996, ApJ, 469, 355

Gerth, E., \& Glagolevskij, Yu. V. 2004, in The A-star Puzzle, ed. J. Zverko, J. Žižňovský, S. J. Adelman, \& W. W. Weiss, Proc. IAU Symp., 224, 629

Gerth, E., \& Glagolevskij, Yu. V. 2001, in Magnetic fields across the Herzsprung-Russel diagram, ed. G. Mathys, S. K. Solanki, \& D. T. Wickramsinghe, Santiago de Chile, 248, 333

Gerth, E., Glagolevskij, Yu. V., \& Scholz, G. 1997, in Stellar Magnetic Fields, ed. Yu. V. Glagolevskij, \& I. I. Romanyuk, Moscow, 67 Jugaku, J., \& Sargent, W. L. W. 1968, ApJ, 151, 259

Khalack, V. R. 2002, Phys. and Kinem. of Cel. Bodies, 18(6), 553

Khalack, V., Zverko, J., \& Žižňovský, J. 2003, A\&A, 403, 179

Khalack, V. R., Khalack, J. N., Shavrina, A.V., \& Polosukhina, N. S. 2001, Astron. Reports, 45, 564

King, J. R., Villarreal, A. R., Soderblom, D. R., et al. 2003, AJ, 125, 1980

Kochukhov, O., Bagnulo, S., Wade, G. A., et al. 2004, A\&A, 414, 613

Kochukhov, O., Piskunov, N., Ilyin, I., et al. 2002, A\&A, 389, 420

Kochukhov, O., \& Piskunov, N. 2002, A\&A, 388, 868

Kupka, F., Piskunov, N. E., Ryabchikova, T. A., et al. 1999, A\&AS, 138,119

Kurucz, R. 1994, CDR(1-23)

Landolfi, M., Landi degl'Innocenti, E., Landi degl'Innocenti, M., \& Leroy, J. L. 1993, A\&A, 272, 285

Landstreet, J. D. 1988, ApJ, 326, 967

Landstreet, J. D. 1982, ApJ, 258, 639

Leone, F., \& Catanzaro, G. 2001, A\&A, 365, 118
Leroy, J. L. 1995, A\&AS, 114, 79

Leroy, J. L., Landolfi, M., \& Landi degl'Innocenti, E. 1996, A\&A, 311,513

Mihalas, D., \& Henshaw, J. L. 1966, ApJ, 144, 25

Monier, R. 1992, A\&A, 263, 175

Raassen, A. J. J., \& Uylings, P. H. M. 1998, A\&A, 340, 300

Ryabchikova, T. A., Piskunov, N. E., Stempels, H. C., Kupka, F., \& Weiss, W. W. 1999, Proc. of the 6th International Colloquium om Atomic Spectra and Oscillator Strengths, Victoria BC, Phys. Scr., $\mathrm{T} 83,162$

Press, W. H., Teukolsky, S. A., Vetterling, W. T., \& Flannery, B. P. 1992, Numerical recipes in C: the art of scientific computing, 2nd ed. (Cambridge University Press), 995

Peston, G. W. 1971, ApJ, 164, 309

Peston, G. W. 1969, ApJ, 158, 243

Sandwell, D. T. 1987, Geophys. Res. Lett., 2, 139

Schaller, G., Schaerer, D., Meynet, G., \& Maeder, A. 1992, A\&AS, 96,269

Stibbs, D. W. N. 1950, MNRAS, 110, 395

Wade, G. A., Bagnulo, S., Kochukhov, O., et al. 2001, A\&A, 374, 265

Wade, G. A., Donati, J.-F., Landstreet, J. D., \& Shorlin, S. L. S. 2000a, MNRAS, 313, 823

Wade, G. A., Donati, J.-F., Landstreet, J. D., \& Shorlin, S. L. S. 2000b, MNRAS, 313, 851

Wolff, S. C. 1978, PASP, 90,412

Wolff, S. C. 1967, ApJS, 15, 21

Wolff, S. C., \& Bonsack, W. K. 1972, ApJ, 176, 425

Wolff, S. C., \& Wolff, R. J. 1971, AJ, 76, 422 